\title{
Spinning gravitating objects in the effective field theory in the post-Newtonian scheme
}

\author{
Michele Levi ${ }^{a, b}$ and Jan Steinhoff ${ }^{c, d}$ \\ ${ }^{a}$ Université Pierre et Marie Curie-Paris VI, CNRS-UMR 7095, \\ Institut d'Astrophysique de Paris, 98 bis Boulevard Arago, 75014 Paris, France \\ ${ }^{b}$ Sorbonne Universités, Institut Lagrange de Paris, \\ 98 bis Boulevard Arago, 75014 Paris, France \\ ${ }^{c}$ Max-Planck-Institute for Gravitational Physics (Albert-Einstein-Institute), \\ Am Mühlenberg 1, 14476 Potsdam-Golm, Germany \\ ${ }^{d}$ Centro Multidisciplinar de Astrofisica, Instituto Superior Tecnico, Universidade de Lisboa, \\ Avenida Rovisco Pais 1, 1049-001 Lisboa, Portugal \\ E-mail: michele.levi@upmc.fr, jan.steinhoff@aei.mpg.de
}

ABSTRACT: We introduce a formulation for spinning gravitating objects in the effective field theory in the post-Newtonian scheme in the context of the binary inspiral problem. We aim at an effective action, where all field modes below the orbital scale are integrated out. We spell out the relevant degrees of freedom, in particular the rotational ones, and the associated symmetries. Building on these symmetries, we introduce the minimal coupling part of the point particle action in terms of gauge rotational variables, and construct the spin-induced nonminimal couplings, where we obtain the leading order couplings to all orders in spin. We specify the gauge for the rotational variables, where the unphysical degrees of freedom are eliminated already from the Feynman rules, and all the orbital field modes are integrated out. The equations of motion of the spin can be directly obtained via a proper variation of the action, and Hamiltonians may be straightforwardly derived. We implement this effective field theory for spin to derive all spin dependent potentials up to next-to-leading order to quadratic level in spin, namely up to the third post-Newtonian order for rapidly rotating compact objects. In particular, the proper next-to-leading order spin-squared potential and Hamiltonian for generic compact objects are also derived. For the implementations we use the nonrelativistic gravitational field decomposition, which is found here to eliminate higher-loop Feynman diagrams also in spin dependent sectors, and facilitates derivations. This formulation for spin is thus ideal for treatment of higher order spin dependent sectors.

Keywords: Classical Theories of Gravity, Effective field theories, Renormalization Regularization and Renormalons, Black Holes

ARXIV EPRINT: 1501.04956 


\section{Contents}

1 Introduction 1

2 Setup of EFT for gravitating spinning objects 4

2.1 Setup and goal 4

2.2 Degrees of freedom 6

$\begin{array}{lll}2.3 & \text { Symmetries } & 7\end{array}$

3 Formulation of EFT for spin $\quad \mathbf{8}$

3.1 EFT with the worldline spin as a further DOF 8

$\begin{array}{ll}3.2 & \text { Unfixing the gauge of the rotational variables }\end{array}$

$\begin{array}{lll}3.3 & \text { Extra term from minimal coupling } & 13\end{array}$

4 EFT for spin: nonminimal couplings $\quad \mathbf{1 5}$

$\begin{array}{lll}\text { 4.1 Construction of spin nonminimal couplings } & 15\end{array}$

$\begin{array}{lll}4.2 & \text { Nonminimal couplings to all orders in spin } & 19\end{array}$

5 Integrating out the orbital scale $\quad 21$

$\begin{array}{ll}5.1 \text { Tetrad field gauge } & 22\end{array}$

5.2 Fixing the gauge of the rotational variables 23

$\begin{array}{lll}5.3 & \text { Feynman rules } & 27\end{array}$

$\begin{array}{ll}5.4 \text { EOM of the spin } & 28\end{array}$

$6 \quad$ Spin potentials to NLO $\quad 29$

$\begin{array}{lll}6.1 \text { LO spin sectors } & 30\end{array}$

$\begin{array}{lll}\text { 6.1.1 LO spin-orbit sector } & 30\end{array}$

6.1.2 LO quadratic in spin sectors 31

$\begin{array}{lll}6.2 & \text { NLO spin-orbit sector } & 32\end{array}$

$\begin{array}{lll}6.3 & \text { NLO spin1-spin2 sector } & 35\end{array}$

$\begin{array}{lll}6.4 & \text { NLO spin-squared sector } & 38\end{array}$

$\begin{array}{lll}7 & \text { Conclusions } & 41\end{array}$

\section{Introduction}

The anticipated direct detection of gravitational waves (GWs) may be realized soon with the upcoming operation of second-generation ground-based interferometers, such as the twin Advanced LIGO [1] detectors in the US, Advanced Virgo [2] in Europe, and in a few years also KAGRA [3] in Japan. This will open a new era of observational gravitational wave astronomy, where also space-based detectors, such as eLISA [4, 5] are planned to 
extend the observed frequency range to the low frequency band. Binaries of compact objects are the most promising sources in the accessible frequency band of such experiments. The post-Newtonian (PN) approximation of General Relativity stands out among the various and complementary approaches to model these systems, as it enables to treat analytically the inspiral phase of their evolution [6].

The search for GW signals from such sources employs the matched-filtering technique, and thus accurate theoretical template waveforms are crucial to obtain a successful detection. Even relative high order PN corrections, such as the fourth PN (4PN) order, have an impact on the waveform templates for the binary inspiral, and further they are required to gain information about the inner structure of the components of the binary [7]. Moreover, astrophysical observations indicate that such black hole components have near extreme spin [8]. Hence, PN spin effects for rapidly rotating compact objects, which first appear at 1.5PN order, should be obtained at least to $4 \mathrm{PN}$ order, which was recently completed in the non-spinning case [9].

Several efforts have been made in recent years to push ahead the formulation for gravitating spinning objects in the context of the binary inspiral problem. An action formalism plays a central role in the various approaches, building in particular on the seminal works in [10] and [11] for flat and curved spacetime, respectively, and see also section 11 of [6] for a review of spinning compact binaries for gravitational radiation. The self-contained Effective Field Theory (EFT) approach for the binary inspiral as introduced in $[12,13]$ for non-spinning objects seems then to provide a solid path to obtain such a formulation. In the EFT formulation manifest power counting in the small expansion parameter (here $v \ll c=1$ ) is achieved by performing a decomposition of the gravitational field at the level of the action into modes with definite scaling properties, followed by integrating out the off-shell modes [12].

The EFT approach provides a systematic methodology to construct the action to arbitrarily high accuracy, in terms of operators with Wilson coefficients ordered by relevance, which is indispensable beyond the point-mass approximation. In that respect it should be pointed out, that even just the point-mass approximation, which past work was using, is naturally incorporated already in the EFT framework. It should also be noted that some effective action with derivative expansion to model finite size effects was already discussed in [14] in the context of alternative theories of gravity. The EFT approach also provides a natural framework to handle the regularization required for higher order corrections in the PN approximation within the standard renormalization scheme. It formulates the perturbative calculation efficiently by applying the standard tools from Quantum Field Theory, such as Feynman diagrams (a related basic diagrammatic expansion was used already in [15]). Consequently, the EFT approach then benefits from existent developed Feynman integral calculus at its disposal. For spinning objects such EFT techniques were first used in [16], and revised in [17], where eventually a Routhian approach from [18] was adopted.

Our goal in this work is indeed to obtain an EFT formulation for gravitating spinning objects for the binary inspiral problem, building on [12, 13], and on several observations made in a series of works, mainly [19-21] and [22]. The essential obstacle that one has to deal with in extending the formulation from a gravitating point-mass to a spinning object 
in terms of an EFT point particle approach is just the intrinsic conflict between the actual spinning object, which must be extended for its rotational velocity not to be superluminal, and its view as a point particle. The elusive notion of a 'center', which would serve as a reference point within the object, in relativistic physics, similar to the center of mass in Newtonian physics, is the origin of ambiguities in the description of relativistic spinning objects. Ever since the first treatment in 1959 of the leading order (LO) PN correction, which involves spin, in the spin-orbit sector, the essential choice of such a center has been a puzzling issue, see Tulczyjew's paper and errata in [23].

In this work we aim at an effective action, that incorporates the essential requirement, that all field modes below the orbital scale are integrated out. We aim to attain accuracy at the $4 \mathrm{PN}$ order for rapidly rotating compact objects, and indeed the formulation in this paper holds as it stands to this high PN order, and it may hold until dissipative effects start to play a role as of the $5 \mathrm{PN}$ order [24]. Here, we spell out the relevant degrees of freedom (DOFs), in particular the rotational ones, and most importantly the associated symmetries. Building on these symmetries, we start with the minimal coupling part of the point particle action, stressing the role of the worldline spin as a further worldline rotational DOF. We proceed to construct the spin-induced nonminimal couplings, where we obtain the LO couplings to all orders in spin. We then introduce the gauge freedom of the rotational variables into the action, and express it in terms of gauge rotational variables. Again, this spin gauge invariance was not addressed previously in the action. From introducing this spin gauge freedom we get that the minimal coupling part of the spin in the action, would contribute to the finite size effects, which is just the manifestation of the aforementioned conflict between the actual spinning extended object and its view as a point particle. We then fix a canonical gauge for the rotational variables, where the unphysical DOFs are eliminated already from the Feynman rules, and all the orbital field modes are conveniently integrated out.

The equations of motion (EOM) of the spin are then directly obtained via a proper variation of the action, where they take on a simple form. The corresponding Hamiltonians are also straightforwardly obtained from the potentials, derived via this formulation, due to the canonical gauge fixing. We implement this EFT formulation for spin to derive all spin dependent potentials up to next-to-leading order (NLO) to quadratic level in spin, i.e. up to the $3 \mathrm{PN}$ order for rapidly rotating compact objects. In particular, the proper nextto-leading order spin-squared potential and Hamiltonian for generic compact objects are also derived. For the implementations we use the nonrelativistic gravitational (NRG) field decomposition $[25,26]$, which is found here to eliminate higher-loop Feynman diagrams also for spin dependent sectors, and facilitates derivations. Hence, with the simple EOM of the spin, and the additional advantageous usefulness of the Hamiltonian for the straightforward obtainment of gauge-invariant quantities, and for implementations within the effective onebody formulation [27], the EFT formulation for spin here is ideal for treatment of higher order spin dependent sectors. Indeed, the application of the EFT formulation for spin presented here has led to the completion of the spin dependent conservative sector up to the $4 \mathrm{PN}$ order in the recent works [28, 29], and [30], which obtained the LO cubic and quartic in spin, NNLO spin-orbit, and NNLO spin-squared sectors, respectively. 
The outline of the paper is as follows. In section 2 we present the setup and goal of our EFT formulation for gravitating spinning objects, and detail the relevant DOFs, and the associated symmetries. In section 3 we start by presenting the minimal coupling part of the action, and then express it in terms of rotational gauge variables, which yields an extra term from minimal coupling. In section 4 we construct the spin-induced nonminimal coupling part of the action, where we obtain the LO couplings to all orders in spin. In section 5 we fix all ingredients in order to integrate out the orbital field modes: we disentangle the tetrad field from the worldline tetrad, we fix the gauge of the tetrad field and of the rotational variables, and present the resulting Feynman rules. We also discuss how the EOM of the spin are then directly obtained after the orbital modes have been integrated out. In section 6 we implement this EFT for spin to derive all spin dependent potentials and Hamiltonians up to NLO to quadratic level in spin, i.e. up to the 3PN order for rapidly rotating compact objects. In section 7 we summarize our main conclusions.

Throughout this paper we use $c \equiv 1, \eta_{\mu \nu} \equiv \operatorname{Diag}[1,-1,-1,-1]$, and the convention for the Riemann tensor is $R_{\nu \alpha \beta}^{\mu} \equiv \partial_{\alpha} \Gamma_{\nu \beta}^{\mu}-\partial_{\beta} \Gamma_{\nu \alpha}^{\mu}+\Gamma_{\lambda \alpha}^{\mu} \Gamma_{\nu \beta}^{\lambda}-\Gamma_{\lambda \beta}^{\mu} \Gamma_{\nu \alpha}^{\lambda}$. Greek letters denote indices in the global coordinate frame, lowercase Latin letters from the beginning of the alphabet denote indices in the local Lorentz frame, and upper case Latin letters from the beginning of the alphabet denote the worldline tetrad frame. All indices run from 0 to 3 , while spatial tensor indices from 1 to 3 , are denoted with lowercase Latin letters from the middle of the alphabet. Square brackets on indices denote that they are in the worldline tetrad frame. Uppercase Latin letters from the middle of the alphabet denote particle labels. The scalar triple product appears here with no brackets, i.e. $\vec{a} \times \vec{b} \cdot \vec{c} \equiv(\vec{a} \times \vec{b}) \cdot \vec{c}$.

\section{Setup of EFT for gravitating spinning objects}

\subsection{Setup and goal}

We begin by recalling the general setup of an EFT for the binary inspiral problem in terms of a tower of EFTs, building on $[12,13]$. The binary inspiral problem involves two intermediate scales below the radiation wavelength scale, $\lambda$, which are the scale of internal structure of each of the compact components of the binary, $r_{s} \sim m$, where $m$ is the mass of the compact object, and the orbital separation scale, $r$. It holds that $r \sim r_{s} / v^{2} \sim \lambda v$, where $v$ is the typical nonrelativistic orbital velocity at the inspiral phase, that is $v \ll 1$. Hence, there is a hierarchy of scales in the binary inspiral problem, which makes it ideal for an EFT treatment. We note that we consider here gravitating objects, which are in general spinning.

Therefore, to obtain an EFT describing the radiation from the binary, one should proceed in two stages:

1. First, we should have an EFT that removes the scale of the compact objects, $r_{s}$, from the purely gravitational action of the isolated compact object, which is just the Einstein-Hilbert action

$$
S\left[g_{\mu \nu}\right]=-\frac{1}{16 \pi G} \int d^{4} x \sqrt{g} R
$$


We integrate out the strong field modes, $g_{\mu \nu}^{s}$, where $g_{\mu \nu} \equiv g_{\mu \nu}^{s}+\bar{g}_{\mu \nu}$, by writing down an effective action containing the most general set of worldline operators consistent with the symmetries of the theory. According to the decoupling theorem [31] the effective action can be expressed by introducing an infinite tower of worldline operators $O_{i}(\sigma)$, such that

$$
S_{\text {eff }}\left[y^{\mu}, e_{A}^{\mu}, \bar{g}_{\mu \nu}\right]=-\frac{1}{16 \pi G} \int d^{4} x \sqrt{\bar{g}} R\left[\bar{g}_{\mu \nu}\right]+\underbrace{\sum_{i} C_{i} \int d \sigma O_{i}(\sigma)}_{S_{p p} \equiv \text { point particle action }},
$$

where $y^{\mu}$ and $e_{A}^{\mu}$ are the particle worldline coordinate and worldline tetrad degrees of freedom (DOFs), discussed in the following section. All UV dependence shows up only in the Wilson coefficients $C_{i}\left(r_{s}\right)$ in the point particle action, $S_{\mathrm{pp}}$, and the worldline operators $O_{i}(\sigma)$ must respect the symmetries of the relevant DOFs at this scale. In sections 2.2 and 2.3 below we elaborate on the degrees of freedom and the symmetries, considering gravitating spinning objects.

We note that a spinning point particle is characterized by two parameters, its mass, $m$, and spin length, $S^{2}$, to be defined in sections 3.1 and 4.1. Yet, since $S \lesssim m^{2} \sim r_{s}^{2}$, then indeed $r_{s}$ is the only scale in the full theory. In addition, dissipative effects from the absorption of gravitational waves by the compact objects, as considered in e.g. [24], which modify the mass and spin of the objects, enter only as of the 5PN order. Hence, the mass and spin length can be considered as constant for all relevant implementations.

2. The following EFT in the tower should have the orbital scale of the binary removed. The metric field is again decomposed into the modes

$$
\bar{g}_{\mu \nu} \equiv \eta_{\mu \nu}+\underbrace{H_{\mu \nu}}_{\text {orbital }}+\underbrace{\widetilde{h}_{\mu \nu}}_{\text {radiation }}
$$

and we note that

$$
\partial_{t} H_{\mu \nu} \sim \frac{v}{r} H_{\mu \nu}, \quad \partial_{\rho} \widetilde{h}_{\mu \nu} \sim \frac{v}{r} \widetilde{h}_{\mu \nu},
$$

whereas

$$
\partial_{i} H_{\mu \nu} \sim \frac{1}{r} H_{\mu \nu}
$$

This EFT of the binary, which is regarded now as a single composite object, is obtained by explicitly integrating out the field modes below the orbital scale, $H_{\mu \nu}$. Starting from an effective action of a binary, given by

$$
S_{\mathrm{eff}}\left[y_{1}^{\mu}, y_{2}^{\mu}, e_{(1) A}^{\mu}, e_{(2) A}^{\mu}, \bar{g}_{\mu \nu}\right]=-\frac{1}{16 \pi G} \int d^{4} x \sqrt{\bar{g}} R\left[\bar{g}_{\mu \nu}\right]+S_{(1) \mathrm{pp}}+S_{(2) \mathrm{pp}},
$$

the effective action of the composite object is defined by the functional integral

$$
e^{i S_{\mathrm{eff}(\mathrm{composite)}}\left[y^{\mu}, e_{A}^{\mu}, \widetilde{h}_{\mu \nu}\right]} \equiv \int \mathcal{D} H_{\mu \nu} e^{i S_{\mathrm{eff}}\left[y_{1}^{\mu}, y_{2}^{\mu}, e_{(1)}^{\mu}, e_{(2)}^{\mu}, \bar{g}_{\mu \nu}\right]}
$$


considering the classical limit, i.e. evaluating the relevant Feynman diagrams in the tree level approximation. Here $y^{\mu}$ and $e_{A}^{\mu}$ are the worldline coordinate and tetrad, i.e. $\eta^{A B} e_{A}{ }^{\mu}(\sigma) e_{B}{ }^{\nu}(\sigma)=\eta^{\mu \nu}+\widetilde{h}^{\mu \nu}$, of the composite particle, respectively.

To obtain the final EFT in the tower, an EFT of radiation, the field DOFs should all be integrated out. Therefore, in general one has to proceed to a third stage, where also the radiation modes, $\widetilde{h}_{\mu \nu}$, are integrated out. Yet, in the conservative sector, where no radiation modes are present, and from which the conservative dynamics is inferred, the EFT construction process ends after the aforementioned two stages, that is after having integrated out the field modes below the orbital scale.

Indeed, in this paper we focus on the imperative two stage process for the conservative sector, where the end goal of this process should be an effective action, i.e. an action without any remaining orbital scale field DOFs. Naturally, this also involves eliminating all unphysical DOFs from the action, in particular those associated with the rotational DOFs, see section 3 in [22]. By definition, e.g. in eqs. (2.3) and (2.7), an effective action should not contain any remaining field DOFs of modes of the scale, which it removes. These should all be integrated out.

It should also be noted that this construction of the EFT, starting from the scale of the internal structure of the compact objects, $r_{s}$, should be supplemented below this scale for compact stars, rather than just black holes. This becomes relevant, when nonminimal couplings should be taken into account, and we comment on that in section 4 .

\subsection{Degrees of freedom}

We should specify and keep track of our degrees of freedom in the process of constructing the EFTs. We should consider here three kinds of DOFs:

1. The gravitational field. For the effective action in eq. (2.2) we have the field DOFs in the purely gravitational action, and in the non-spinning point particle actions, simply represented by the metric $g_{\mu \nu}(x)$ (the overbar notation of the metric is dropped here and henceforth). For the point particle actions beyond the mass monopole, which also involve the spins, the tetrad field, $\eta^{a b} \tilde{e}_{a}^{\mu}(x) \tilde{e}_{b}^{\nu}(x)=g^{\mu \nu}(x)$, which couples to the multipoles of the objects, also represents the field DOFs. After gauge fixing the purely gravitational action, and the tetrad field, both the metric and the tetrad fields are left with 6 DOFs.

2. The particle worldline coordinate. $y^{\mu}(\sigma)$ is a function of an arbitrary affine parameter $\sigma$. The time coordinate is used to fix the gauge of the affine parameter, and we have the 3 DOFs, giving the position of the particle. The particle worldline position does not in general coincide with the 'center' of the object, that is the reference point within the actual extended object. The 'center' is uniquely defined in Newtonian physics, but not in relativity theory.

3. The particle worldline rotating DOFs. Initially, we consider the worldline tetrad, an orthonormal frame $\eta^{A B} e_{A}^{\mu}(\sigma) e_{B}^{\nu}(\sigma)=g^{\mu \nu}$, localized on the particle worldline, connecting the body-fixed and general coordinate frames. From this tetrad we define the 
worldline angular velocity $\Omega^{\mu \nu}(\sigma)$, and then we add its conjugate, the worldline spin, $S_{\mu \nu}(\sigma)$, as a further DOF. We then have $6+6$ DOFs. The field DOFs, represented by the tetrad field, which satisfies $\tilde{e}_{a}^{\mu}(y(\sigma))=\Lambda_{a}{ }^{A}(\sigma) e_{A}{ }^{\mu}(\sigma)$, are then disentangled from the worldline tetrad DOFs, such that we are left with the worldline Lorentz matrices DOFs, $\eta^{A B} \Lambda_{A}{ }^{a}(\sigma) \Lambda_{B}{ }^{b}(\sigma)=\eta^{a b}$, and the conjugate worldline spin, $S_{a b}(\sigma)$, projected to the local frame. After gauge fixing the rotational DOFs, we are left only with the $3+3$ physical DOFs.

\section{$2.3 \quad$ Symmetries}

The aforementioned degrees of freedom should be coupled in all possible ways allowed by the symmetries of the problem in order to construct the effective action. The following symmetries should then be considered:

1. General coordinate invariance, and in particular parity invariance is also included, which holds for macroscopic objects in General Relativity, and is relevant for nonminimal couplings in the point particle action, see section 4.

2. Worldline reparametrization invariance. This is used to construct the minimal coupling as well as the non minimal coupling parts of the point particle action, see sections 3.1 and 4, respectively.

3. Internal Lorentz invariance of the local frame field. We use the $3+3$ DOFs of local Lorentz transformations to fix the gauge of the tetrad field, which in general has 16 DOFs, such that it is represented by the 10 DOFs of the metric (before gauge fixing), see section 5.1.

4. $\mathrm{SO}(3)$ invariance of the body-fixed spatial triad, $e_{[i]}^{\mu}$, consisting of the 3 spacelike vectors. This follows from the 3 rotational DOFs to orient the massive particle in space in the body-fixed frame. In consequence, the worldline spin DOFs are $\mathrm{SO}(3)$ tensors in the body-fixed frame, which is also relevant for nonminimal couplings in the point particle action, see section 4 . This is also discussed in section 3.2 in relation with the spin gauge invariance.

5. Spin gauge invariance, that is an invariance under the choice of a completion of the body-fixed spatial triad through a timelike vector. This is a gauge of the rotational variables, i.e. of the worldline tetrad and of the worldline spin. It is considered in section 3.2, and further discussed in section 5.2.

6. We assume that the isolated object has no intrinsic permanent multipole moments beyond the mass monopole and the spin dipole. This is used in sections 3.1 and 4 . Permanent multipole moments may be included through constant $\mathrm{SO}(3)$ tensors. Yet, recall that mass and spin are conserved for isolated objects, but higher multipoles are not.

We stress that time-reversal symmetry is not assumed here, but instead terms which violate it are shown not to contribute at the considered order, see also section 4 . 


\section{$3 \quad$ Formulation of EFT for spin}

\subsection{EFT with the worldline spin as a further DOF}

First, we briefly review the essential basic definitions as in, e.g. section III of [20]. We start by considering the worldline tetrad, an orthonormal frame $e_{A}^{\mu}(\sigma)$, localized on the particle worldline, connecting the body-fixed and general coordinate frames, such that $\eta^{A B} e_{A}^{\mu} e_{B}^{\nu}=g^{\mu \nu}$ with $\eta^{A B} \equiv \operatorname{diag}[1,-1,-1,-1]$ the flat spacetime Minkowski metric. We recall that the reciprocal tetrad is defined by $e^{\mu A} \equiv \eta^{A B} e_{B}^{\mu}$. The projections of any tensor onto the tetrad frame, and the converse projection onto the coordinate frame are then defined as, e.g. for a vector, $V_{A} \equiv e_{A}^{\mu} V_{\mu}$, and $V_{\mu} \equiv e_{\mu}^{A} V_{A}$, respectively.

We proceed to define the antisymmetric angular velocity tensor by

$$
\Omega^{\mu \nu} \equiv e_{A}^{\mu} \frac{D e^{A \nu}}{D \sigma},
$$

where $D / D \sigma$ is the covariant derivative with respect to the worldline parameter $\sigma$, and this is a generalization of the flat spacetime definition given by $\Omega^{a b} \equiv \Lambda_{A}^{a} \frac{d \Lambda^{A b}}{d \sigma}[10,11]$. Considering the degrees of freedom and symmetries of the problem, noted in the previous section, the point particle Lagrangian should be a function of the coordinate velocity, $u^{\mu} \equiv d y^{\mu} / d \sigma$, the angular velocity from eq. (3.1), and the metric, that is $L_{\mathrm{pp}}\left[u^{\mu}, \Omega^{\mu \nu}, g_{\mu \nu}\right]$, where the dependence in the metric is extended beyond minimal coupling to include the Riemann tensor and further covariant derivatives. The spin is then defined as the conjugate to the angular velocity, i.e.

$$
S_{\mu \nu} \equiv-2 \frac{\partial L}{\partial \Omega^{\mu \nu}}
$$

The minus sign in this definition is chosen to give the correct form in the nonrelativistic limit. It is then beneficial to construct the Lagrangian with the spin as a further worldline DOF since it makes sense to utilize the spin dipole moment, sourcing the gravitons, similar to the mass monopole, as a classical source on the worldline. Another advantage is then that the spin becomes an independent variational variable, and the equations of motion (EOM) of the spin are then directly and conveniently obtained via an appropriate variation of the effective action [22].

Therefore, the point particle action from eq. (2.2) can be written as

$$
S_{\mathrm{pp}}=\int d \sigma\left[-m \sqrt{u^{2}}-\frac{1}{2} S_{\mu \nu} \Omega^{\mu \nu}+L_{\mathrm{SI}}\left[u^{\mu}, S_{\mu \nu}, g_{\mu \nu}\left(y^{\mu}\right)\right]\right],
$$

where the first two terms are just the point-mass and rotational minimal couplings retained from flat spacetime $[10,11]$, which are inferred from reparametrization invariance. $L_{\mathrm{SI}}$ stands for the nonminimal coupling part of the action, which according to the symmetries spelled out in section 2.3 contains only spin-induced multipoles, and as will be further illustrated in section 4 , only depends on the worldline DOFs $u^{\mu}$ and $S_{\mu \nu}$. The conjugate to the 4 -velocity $u^{\mu}$ is the linear momentum, given by

$$
p_{\mu} \equiv-\frac{\partial L}{\partial u^{\mu}}
$$


Clearly, it is Lagrangian dependent and is modified as higher multipoles, i.e. nonminimal couplings, are introduced, and we have

$$
p^{\mu}=m \frac{u^{\mu}}{\sqrt{u^{2}}}+\mathcal{O}\left(S^{2}\right) .
$$

For an isolated compact object the linear momentum $p^{\mu}$ can be obtained from surface integrals at spatial infinity. In this case finite size effects are not taken into account, and the mass is matched as $m^{2}=p_{\mu} p^{\mu}$.

We note that we can also express the rotational minimal coupling term, using the spin projected to the body-fixed frame, where the spin is a permanent multipole moment. Indeed, the components of the spin in this frame are constant, which can be seen most directly using the EOM following from the action in eq. (3.3). Using the Ricci rotation coefficients, defined by

$$
\omega_{\mu}^{a b} \equiv e^{b}{ }_{\nu} D_{\mu} e^{a \nu}
$$

it holds that

$$
\frac{1}{2} S_{\mu \nu} \Omega^{\mu \nu}=\frac{1}{2} S_{A B} \omega_{\mu}^{A B} u^{\mu} .
$$

We shall see that only the spatial $\mathrm{SO}(3)$ components in the body-fixed frame are nonvanishing here. Considering the scalar mass monopole from eq. (3.3), and this form, where the spin dipole is also represented as a constant antisymmetric $\mathrm{SO}(3)$ tensor, we shall be able to construct the nonminimal coupling part of the action in a rather straightforward manner, as will be detailed in section 4 .

As we are working in an action approach, there is no impediment to the implementation of gauge constraints on the rotational DOFs. Moreover, as we shall see these gauge constraints should be implemented at the level of the point particle action in order to ultimately arrive at an effective action without any remaining orbital scale field degrees of freedom. We shall also see in the following section 3.2, that in order to arrive at a generic point particle action, where the gauge of the rotational variables is not fixed, we should initially implement the covariant gauge. Yet it is crucial to point out that in the point particle Lagrangian in eq. (3.3), we have both DOFs of the angular velocity and of the spin, and therefore it is necessary to implement gauge fixing both on the worldline tetrad DOFs and on the spin DOFs, rather than only on the latter ones. We shall explicitly see in section 5.2, that we cannot obtain an effective action formulated with the worldline spin, if the gauge of the spin is fixed without gauge fixing its conjugate DOFs. These are principal statements in this paper.

\subsection{Unfixing the gauge of the rotational variables}

As we noted in section 2.3 there is a spin gauge freedom in the choice of a timelike vector for the worldline tetrad. This is a choice of a 'center' point within the spinning object, which must have a finite size due to its spin. This gauge is fixed using some spin supplementary conditions (SSC), corresponding to a gauge choice of the timelike basis vector for the worldline tetrad. The covariant SSC by Tulczyjew [32], given by

$$
S_{\mu \nu} p^{\nu}=0,
$$


where $p^{\nu}$ is the linear momentum from eq. (3.4), is the only SSC, for which existence and uniqueness of a corresponding 'center' were proven rigorously in general relativity [33, 34]. Together with the corresponding gauge condition on the worldline tetrad timelike basis vector, which reads

$$
e_{[0]}^{\mu}=p^{\mu} / \sqrt{p^{2}}
$$

we see that this gauge is equivalent to the requirement that the spin in the body-fixed frame, as in eq. (3.7), is a spatial $\mathrm{SO}(3)$ tensor.

General coordinate invariance is an important symmetry of the effective action, and it makes sense to specify the point particle action for this covariant SSC. However, we also expect to have a spin gauge symmetry in the effective action, such that indeed there is gauge freedom in the choice of the timelike vector for the worldline tetrad. This gauge symmetry was not addressed in previous work, which approached to extend the EFT formulation for spinning objects. Therefore, we start out with the covariant gauge, having a covariant theory at hand, and then transform to a generic gauge, thus introducing the spin gauge freedom into the action. Hence, we illustrate a procedure for directly constructing an action, which is manifestly invariant under both coordinate and rotational variables gauge transformations.

We are approaching the change of a spin gauge from a new perspective. We are effectively applying a boost to the body-fixed tetrad, and then we see how the rotational minimal coupling term $\frac{1}{2} S_{\mu \nu} \Omega^{\mu \nu}$ in the action in eq. (3.3) is affected. This approach is in fact suggested by the EFT philosophy since an important ingredient in the EFT setup is the $\mathrm{SO}(3)$ invariance of the worldline spatial triad, rather than $\mathrm{SO}(1,3)$ Lorentz invariance of the worldline tetrad. Since the velocity of the particle is already set as the time derivative of its position coordinate, the additional boost degrees of freedom of the internal $\operatorname{SO}(1,3)$ indices of the worldline tetrad are actually redundant gauge ones. Only the orientation of the particle is physical, and can be described by an internal $\mathrm{SO}(3)$ group. Then the action should be formulated in terms of $e_{[i]}{ }^{\mu}$, which possess $\mathrm{SO}(3)$ indices $i$, without the timelike basis vector $e_{[0]}^{\mu}$. Hence we have the idea that a fixation of $e_{[0]}{ }^{\mu}$ should be connected to the gauge choice of the spin variable or SSC. We are going to show that this is indeed the case. We therefore connect different choices for $e_{[0]}{ }^{\mu}$ by effectively boosting $e_{A}{ }^{\mu}$ in the following.

Consider a boost in the 4 -dimensional covariant form. It is given by

$$
L_{b}^{a}(q, w) \equiv \delta^{a}{ }_{b}+2 q^{a} w_{b}-\frac{\left(q^{a}+w^{a}\right)\left(q_{b}+w_{b}\right)}{1+q w},
$$

where $q w \equiv q_{a} w^{a}$, and $q^{a} q_{a}=w^{a} w_{a}=1$, i.e. $q_{a}, w_{a}$ are timelike unit 4-vectors. We will now make use of the definition in eq. (3.10) with general coordinate indices instead of Lorentz indices. Then $L_{\nu}^{\mu}(q, w)$ is strictly speaking not a Lorentz transformation, but it is taken as the tensor projected by the appropriate tetrad onto the coordinate frame, see e.g. [35]. We can then transform $e^{A \mu}$ from a gauge condition

$$
e_{A \mu} q^{\mu}=\eta_{[0] A} \Leftrightarrow e_{[0] \mu}=q_{\mu},
$$

to the condition

$$
\hat{e}_{A \mu} w^{\mu}=\eta_{[0] A} \Leftrightarrow \hat{e}_{[0] \mu}=w_{\mu}
$$


with the help of the transformation

$$
\hat{e}^{A \mu}=L_{\nu}^{\mu}(w, q) e^{A \nu} .
$$

The algebraic properties of $L^{\mu}{ }_{\nu}(q, w)$ then guarantee that

$$
\eta_{A B} \hat{e}^{A \mu} \hat{e}^{B \nu}=g^{\mu \nu},
$$

if the analogous relation holds for $e^{A \mu}$.

Further, notice that from eqs. (3.11) and (3.12) we have

$$
\begin{aligned}
\frac{D q^{\mu}}{D \sigma} & =-\Omega^{\mu \nu} q_{\nu}, \\
\frac{D w^{\mu}}{D \sigma} & =-\hat{\Omega}^{\mu \nu} w_{\nu},
\end{aligned}
$$

where similarly to eq. (3.1), we have $\hat{\Omega}^{\mu \nu} \equiv \hat{e}_{A} \mu \frac{D \hat{e}^{A \nu}}{D \sigma}$. For the angular velocity we get

$$
\Omega^{\mu \nu}=L_{\rho}^{\mu}(q, w) L_{\sigma}^{\nu}(q, w) \hat{\Omega}^{\rho \sigma}+L_{\rho}^{\mu}(q, w) \frac{D L^{\nu \rho}(q, w)}{D \sigma},
$$

and since it holds that

$$
L_{\rho}^{\mu}(q, w) \frac{D L^{\nu \rho}(q, w)}{D \sigma}=2 \frac{D w^{\mu}}{D \sigma} q^{\nu}-\frac{2 w^{\mu} q^{\nu} q_{\rho}}{1+q w} \frac{D w^{\rho}}{D \sigma}-\frac{q^{\nu}+w^{\nu}}{1+q w} \frac{D\left(q^{\mu}+w^{\mu}\right)}{D \sigma}-(\mu \leftrightarrow \nu),
$$

then the transformation between the covariant angular velocities finally reads

$$
\Omega^{\mu \nu}=\hat{\Omega}^{\mu \nu}+\left[\frac{q^{\mu}+w^{\mu}}{1+q w}\left(\frac{D q^{\nu}}{D \sigma}+\hat{\Omega}^{\nu \rho} q_{\rho}\right)-(\mu \leftrightarrow \nu)\right] .
$$

From this we can easily obtain the effect on the minimal coupling term as

$$
\frac{1}{2} S_{\mu \nu} \Omega^{\mu \nu}=\frac{1}{2}\left(S_{\mu \nu}-\frac{S_{\mu \rho} w^{\rho}}{1+q w} q_{\nu}+\frac{S_{\nu \rho} w^{\rho}}{1+q w} q_{\mu}\right) \hat{\Omega}^{\mu \nu}-\frac{S_{\mu \rho} w^{\rho}}{1+q w} \frac{D q^{\mu}}{D \sigma}
$$

where the SSC $S_{\mu \nu} q^{\nu}=0$ was used. Notice that both the gauge condition on the tetrad, $e_{[0] \mu}=q_{\mu}$, and the SSC should be used at the level of the action in order to transform the minimal coupling term.

The last equation suggests to define a new spin tensor as the prefactor of the transformed angular velocity $\hat{\Omega}^{\mu \nu}$, that is

$$
\hat{S}_{\mu \nu} \equiv S_{\mu \nu}-\frac{S_{\mu \rho} w^{\rho}}{1+q w} q_{\nu}+\frac{S_{\nu \rho} w^{\rho}}{1+q w} q_{\mu} .
$$

If indeed we fix $q^{\mu}=p^{\mu} / \sqrt{p^{2}}$, i.e. we start with Tulczyjew's covariant SSC, then the new spin variable reads

$$
\hat{S}_{\mu \nu}=S_{\mu \nu}-\frac{S_{\mu \rho} w^{\rho}}{\sqrt{p^{2}}+p w} p_{\nu}+\frac{S_{\nu \rho} w^{\rho}}{\sqrt{p^{2}}+p w} p_{\mu}
$$

and satisfies the generic spin supplementary condition

$$
\hat{S}^{\mu \nu}\left(p_{\nu}+\sqrt{p^{2}} \hat{e}_{[0] \nu}\right)=0,
$$


together with the gauge constraint for the tetrad

$$
\hat{e}_{[0] \mu}=w_{\mu},
$$

which provide together the necessary $3+3$ gauge constraints to eliminate the redundant unphysical DOFs in the angular velocity and spin tensors, see section 5.2 for further analysis of specific sensible gauges.

Notice also that the spin transforms as expected under a shift of the 'center' position, $\delta z^{\mu} \equiv \hat{z}^{\mu}-y^{\mu}$, namely as

$$
\hat{S}^{\mu \nu}=S^{\mu \nu}-\delta z^{\mu} p^{\nu}+\delta z^{\nu} p^{\mu} .
$$

By comparing eqs. (3.25) and (3.22) we can read off $\delta z^{\mu}$ as

$$
\delta z^{\mu}=\frac{S^{\mu \rho} w_{\rho}}{\sqrt{p^{2}}+p w} .
$$

Notice that due to the initial covariant SSC it holds that $\delta z^{\mu} p_{\mu}=0$, that is the shift of the 'center' of the object is orthogonal to the linear momentum, and thus indeed spacelike. Hence, the 'center' of the spinning particle is shifted from the worldline position of the particle for a non-covariant gauge of the particle rotating DOFs.

Therefore we see that by choosing a gauge for the tetrad in eq. (3.24), we also specify the choice of SSC in eq. (3.23), the spin variable in eq. (3.22), and the position of the 'center' in eq. (3.26). At this point we have arrived at

$$
\frac{1}{2} S_{\mu \nu} \Omega^{\mu \nu}=\frac{1}{2} \hat{S}_{\mu \nu} \hat{\Omega}^{\mu \nu}-\delta z^{\mu} \frac{D p_{\mu}}{D \sigma} .
$$

Thus, we have introduced spin gauge freedom into the rotational minimal coupling term of the point particle action in eq. (3.3). An alternative construction of the spin gauge symmetry in flat spacetime, based on the canonical formalism, is given in [36].

In order to also introduce this gauge freedom beyond minimal coupling according to eq. (3.3), see section 4 , we need to express our initial spin variable in terms of the generic one in eq. (3.22). We recall that we consider that also the nonminimal coupling terms have been initially constructed for a covariant gauge with a spin variable, satisfying Tulczyjew's covariant SSC. Then we note that from the contraction of eq. (3.22) with $p^{\nu}$, we get that the shift of position of the 'center' can be written as

$$
\delta z^{\mu}=\frac{S^{\mu \rho} w_{\rho}}{\sqrt{p^{2}}+p w}=-\frac{\hat{S}^{\mu \rho} p_{\rho}}{p^{2}},
$$

which leads to

$$
S_{\mu \nu}=\hat{S}_{\mu \nu}-\frac{\hat{S}_{\mu \rho} p^{\rho} p_{\nu}}{p^{2}}+\frac{\hat{S}_{\nu \rho} p^{\rho} p_{\mu}}{p^{2}} .
$$

The transformation to the new generic spin can therefore be written without the $w_{\mu}$ gauge DOFs. This is so since eq. (3.29) is a projection of the generic spin variable $\hat{S}_{\mu \nu}$ onto the spatial hypersurface of the rest frame. This projection removes the gauge DOFs, since all gauges agree in the rest frame, hence the projected spin variable is spin gauge invariant. 
It follows that indeed the action should be constructed using $S_{\mu \nu}$. Recall that the spin variable, which satisfies the covariant SSC, when projected to the body-fixed frame, is a spatial constant $\mathrm{SO}(3)$ tensor.

Finally, the transformation to the generic worldline triad reads

$$
e_{[i]}^{\mu}=\hat{e}_{[i]}^{\mu}-\hat{e}_{[i]}^{\rho} p_{\rho} \frac{p^{\mu}+\sqrt{p^{2}} \hat{e}_{[0]}^{\mu}}{p^{2}+\sqrt{p^{2}} p_{\nu} \hat{e}_{[0]}^{\nu}},
$$

where we have for the temporal component that $e_{[0]}^{\mu}=p^{\mu} / \sqrt{p^{2}}$, and $e_{[i]}{ }^{\mu} p_{\mu}=0$. Notice that only in this relation the new worldline tetrad time vector $\hat{e}_{[0]}{ }^{\nu}=w_{\mu}$ appears explicitly. Hence, the point particle action beyond minimal coupling from eq. (3.3) is constructed from $S_{\mu \nu}$ and $e_{[i]}^{\mu}$, but these are now understood in terms of eqs. (3.29) and (3.30). Actually, eq. (3.30) is not required for spin interactions beyond minimal coupling, but it is needed for dynamical tidal interactions.

Finally, the worldline variables and fields in the point particle action are still taken at $y^{\mu}$. That is, for a non-covariant gauge the worldline position would be shifted from the location $\hat{z}^{\mu}$ of the 'center', which one commonly attributes to $\hat{S}_{\mu \nu}$. Instead, if one chooses to eliminate the covariant derivative in eq. (3.27) at the level of the point particle action by making a shift of the worldline to the location of the 'center', then the worldline variables and fields are implicitly parallel transported to $\hat{z}^{\mu}$. Yet, this is not judicious in order to integrate out all the orbital field modes from the effective action, which can be obtained only if the rotational gauge fixing is implemented at the level of the point particle action. Further, at this stage it is preferable to keep the point particle action in its general form. For further discussion on this point, see also next section 3.3, and section 5.2.

Using the coordinate velocity in the rotational gauge fixing. So far, we have made all derivations regarding the rotational gauge fixing in terms of the linear momentum $p_{\mu}$ for generality. Yet, from eqs. (3.3) and (3.4) we can tell, that the difference $p_{\mu}-m u_{\mu} / u$ is quadratic in the spin, and linear in the field. Then from Tulczyjew SSC, $S_{\mu \nu} p^{\nu}=0$, we can deduce, that this difference may be relevant only as of cubic order in the spin, and in that case, only as of NLO, due to the resulting nonlinear coupling of the field to spin. Therefore, for all current cases of interest, up to the $4 \mathrm{PN}$ order for rapidly rotating compact binaries, we can replace the linear momentum in Tulczyjew SSC with its LO coordinate velocity approximation. In particular, note that this applies to the useful eqs. (3.29), (3.23), (3.27), (3.30), which change the spin variable, fix the redundant temporal spin components, $S^{0 i}$, transform the rotational minimal coupling term, and the worldline tetrad, respectively.

\subsection{Extra term from minimal coupling}

Let us now focus on the second term on the right hand side of eq. (3.27), which involves a covariant derivative of the linear momentum, that is

$$
\frac{\hat{S}^{\mu \nu} p_{\nu}}{p^{2}} \frac{D p_{\mu}}{D \sigma}=-\delta z^{\mu} \frac{D p_{\mu}}{D \sigma} .
$$


This term essentially adds the Thomas precession of the spin in curved spacetime. In flat spacetime the spin does not precess in the absence of a torque, and this term essentially does not contribute. In curved spacetime there is a torque: the force of curvilinear motion on the worldline due to the gravitational field operating on the 'arm', which is the shift from the worldline to the 'center'. Hence, in curved spacetime this term is relevant, and it should be taken into account to all orders in spin. If the covariant derivative of the momentum is eliminated, using the EOM at the level of the point particle action, which corresponds to a redefinition of the position [22,37], it contributes once spin dipole effects are taken into account in the EOM, and thus from eq. (3.31) we see that it first appears in the NLO spin-squared sector. That is, it manifestly contributes as a finite size effect once the spin of the 'particle' is taken into account, as a spinning 'particle' cannot actually be considered a 'particle' anymore, but instead it must have an extended finite size, hence its 'arm' $\sim S / m$, where $S$ is the spin length, defined in eq. (4.5).

It is important to point out the relation of our results to the work by Yee and Bander in [18], which advocated a Routhian approach for the obtainment of the EOM of the spin, and considered up to and including the quadrupolar level. If we consider our eqs. (3.29) for the spin variable, and eq. (3.31) for the extra term from minimal coupling, we can see that they are similar to eqs. (7) and (9) of [18], where their corresponding terms are considered only in the local Lorentz frames, and in the approximation $p_{\mu} \simeq m u^{\mu} / u$.

Yet, it is necessary to make the significant distinction between our formulation here, and their related procedure. Yee and Bander present the change of the spin variable, and the addition of the extra term as an ad hoc procedure to ensure the covariant SSC is satisfied, and to obtain the EOM of the spin via the Poisson brackets, while staying at the so(1,3) level, and not actually implementing the covariant SSC. The work in $[17,38]$ followed their procedure for the treatment of spin, but substituted the worldline acceleration in the extra term similar to eq. (3.31), using the Mathisson-Papapetrou EOM [39-41] for a pole-dipole approximation, given by

$$
\frac{D p_{\mu}}{D \sigma}=-\frac{1}{2} R_{\mu \nu \rho \kappa} u^{\nu} S^{\rho \kappa}
$$

This substitution is a linear approximation in the shift of the worldline, where terms beyond linear in the shift were dropped from the action via the application of the covariant SSC. Thus, an extra Riemann dependent term was introduced, linear in the covariant SSC, which contributes to the NLO spin-squared sector.

First, here we see that since this term originates from minimal coupling, it actually carries no Wilson coefficient. More importantly, it should be stressed that there is no need to require the preservation of the $\mathrm{SSC}$ in the action, and restrict it to the covariant one. The gauge constraints on the worldline tetrad and the SSC should be implemented at the level of the point particle action as was put forward in [19]. Then, using the internal symmetry of the worldline tetrad to incorporate the spin gauge freedom in the point particle action, we obtain here an extra term from minimal coupling. Moreover, here we shall obtain an effective action with the physical $\mathrm{SO}(3)$ rotational variables, and where all orbital field modes are absent. We then conveniently obtain the EOM of the spin via a proper variation of the action, which take on a very simple form, see section 5.4. 


\section{EFT for spin: nonminimal couplings}

In this section we proceed to construct the point particle action for the spinning particle beyond minimal coupling, which accounts for the finite size effects of the gravitating object. These nonminimal couplings carry Wilson coefficients, which encode the internal structure of the object, and should be found by matching the effective theory with the full theory. Here we consider nonminimal couplings of the self-induced multipoles from spin, which contribute as of the $2 \mathrm{PN}$ order for rapidly rotating compact objects. Tidal nonminimal couplings, as considered in [24], contribute only as of the 5PN order, and thus are not of interest for current implementations. It should be noted however that the Wilson coefficients of tidal nonminimal couplings can be large, and tidal interactions are also expected to be important for gravitational wave astronomy.

We first spell out the general considerations for the construction of these nonminimal couplings, building on $[12,13,42,43]$. The point particle action in eq. (3.3) is augmented with higher order operators, where derivatives of the field are added along with higher multipoles. Hence, these new terms are suppressed by powers of the ratio of the scale of the source to the orbital separation, namely they are naturally ordered by their PN relevance. Then, we present the LO nonminimal couplings to all orders in the spin. These were already implemented in [28] by means of the EFT formulation we present here, where the cubic and quartic in spin sectors were fully obtained for generic compact binaries.

As we consider the nonminimal couplings, where the internal structure of the objects starts to play a role, let us comment further on the physics of actual astrophysical extended objects. For generic stars the tower of EFTs presented in section 2.1 starts below the scale $r_{s}$, from a fluid description of matter down to elementary particle interactions. That is, one must add an EFT for matter to eq. (2.1), e.g. an ideal fluid action. The scale of the object can be integrated out explicitly for idealized Newtonian stars [44], which leads to a point-mass action augmented by harmonic oscillator DOFs, corresponding to oscillation modes of the star, which couple to the tidal forces. This is found to approximately hold also for stars in general relativity [45]. It should be noted that when the orbital frequency is in resonance, an infinite number of terms would be needed in eq. (2.2), which indicates that further DOFs should be added to the EFT. This is analogous to resonances in EFTs in particle physics [46], and was overlooked in [12, 42].

\subsection{Construction of spin nonminimal couplings}

We start by discussing the possible spin-induced multipoles. First, from section 2.3 we recall that the action should be parity invariant. Every operator must therefore contain an even number of odd parity tensors, i.e. of the Levi-Civita tensor $\epsilon_{\alpha \beta \gamma \lambda}=\sqrt{-g}[\alpha \beta \gamma \lambda]$, where $g$ is the determinant of $g_{\mu \nu}$, and $[\alpha \beta \gamma \lambda]$ is the totally antisymmetric Levi-Civita symbol with $[0123]=+1$. Therefore, we should consider possible dual tensors, in particular the dual of the spin tensor, given by

$$
* S_{\alpha \beta} \equiv \frac{1}{2} \epsilon_{\alpha \beta \mu \nu} S^{\mu \nu}
$$


Its contraction with the 4-velocity is the spin vector, given by

$$
S^{\mu} \equiv * S^{\mu \nu} \frac{p_{\nu}}{\sqrt{p^{2}}} \simeq * S^{\mu \nu} \frac{u_{\nu}}{\sqrt{u^{2}}} .
$$

Due to the orthogonality of the spin tensor $S^{\mu \nu}$ to $u_{\nu}$ from the covariant SSC, we shall see that the spin vector $S^{\mu}$ is the only combination, with which $u_{\nu}$ can enter the spin-induced multipoles. Notice that $S^{\mu} u_{\mu}=0$, i.e. $S^{\mu}$ is a spacelike vector. Also note that the inverse of the dual is $* * S^{\alpha \beta}=-S^{\alpha \beta}$. Using the SSC, i.e. the orthogonality of $S^{\mu \nu}$ to $u_{\mu}$, at the level of the action, we have then

$$
\begin{aligned}
S_{\mu \alpha} * S^{\alpha \nu} & =-\frac{1}{4} \delta_{\mu}^{\nu} S_{\alpha \beta} * S^{\alpha \beta}=0, \\
S_{\mu \alpha} S^{\alpha} & =0 \\
S_{\mu} S^{\mu} & =-\frac{1}{2} S_{\mu \nu} S^{\mu \nu} \equiv-S^{2},
\end{aligned}
$$

see e.g. [10] for similar identities in flat spacetime, and we defined the spin length $S^{2}$ with the minus sign from the spacelike spin vector.

Now, from the Cayley-Hamilton theorem we expect that higher powers of the spin tensor are dependent. Then, let us examine higher powers of the spin tensor in the sense of matrix multiplication. We have

$$
\begin{aligned}
S^{\alpha}{ }_{\mu} S^{\mu}{ }_{\beta} & =-S^{\alpha} S_{\beta}-S^{2}\left(\delta^{\alpha}{ }_{\beta}-\frac{u^{\alpha} u_{\beta}}{u^{2}}\right), \\
S^{\alpha}{ }_{\mu} S^{\mu}{ }_{\nu} S^{\nu}{ }_{\beta} & =-S^{2} S^{\alpha}{ }_{\beta},
\end{aligned}
$$

where we also note that the square of the spin is symmetric. Indeed, we see that from the last relation we can read off the minimal polynomial of the spin matrix $S^{\alpha}{ }_{\mu}$ as

$$
X(X+i S)(X-i S)=0
$$

which is of degree 3, that is lower than the degree of the characteristic polynomial (4). That means one of the eigenvalues of $S^{\alpha}{ }_{\mu}$ is degenerate. Indeed, for the eigenvalue 0 we have both $S^{\alpha}{ }_{\mu} u^{\mu}=0$, and $S^{\alpha}{ }_{\mu} S^{\mu}=0$. The two remaining eigenvalues of $S^{\alpha}{ }_{\mu}$ are the pure imaginary values $\pm i S$, as expected for the antisymmetric spin matrix. Therefore, the determinant of the spin tensor is zero. A similar analysis for the dual spin tensor leads to no further independent contractions as expected.

In conclusion, from the above analysis we see that the independent combinations of spin that we can use to construct the operators are just the even-parity spin tensor $S^{\mu \nu}$, the odd-parity spin vector $S^{\mu}$, and the even-parity square of the spin tensor $S^{\alpha}{ }_{\mu} S^{\mu}{ }_{\beta}$, where these spin tensors should not be contracted among themselves.

It should be stressed that we implement the covariant SSC at the level of the point particle action, which reduces the number of possible spin-induced multipoles. Yet, since ultimately the spin-induced multipoles should be expressed in terms of the gauge invariant projected spin in eq. (3.29), which is algebraically orthogonal to $u^{\mu}$, then these possibilities would be omitted for all gauge spin variables. 
Further, from section 2.3 we recall the $\mathrm{SO}(3)$ invariance of the body-fixed frame triad. Since we consider our spinning extended body to be a point particle, then there exists a locally inertial frame, which is comoving and in which the spin does not precess [47]. Yet, finite size effects lead to spin precession in this frame. From this comoving, approximately inertial frame, one can move to a corotating one, that is to the body-fixed frame. In the latter, the spin components are constant even in the presence of finite size effects. Moreover, in this locally flat frame we have the $\mathrm{SO}(3)$ invariance as Wigner little group, therefore tensors form $\mathrm{SO}(3)$ irreducible representations [48]. Indeed, in agreement with our analysis of the spin tensor in matrix representation, we find from eq. (4.8), that the spin tensor transforms as a massive vector particle, i.e. like a vector in space. We recall from eqs. (3.3) and (3.7), that the minimal coupling part of the action is given with the scalar mass monopole, and the spin dipole, represented by constant $\mathrm{SO}(3)$ tensors in the body-fixed frame, where the antisymmetric spin is also spatial in the covariant SSC. Hence, the spin-induced higher multipoles should naturally be considered in the body-fixed frame.

Just like in the minimal coupling part of the action, we start from the covariant gauge, where we have for the body-fixed tetrad that $e_{[0]}^{\mu}=u^{\mu} / \sqrt{u^{2}}$, and $e_{[i]}{ }^{\mu} u_{\mu}=0$. Then it is easy to see that in the body-fixed frame it holds for the spin vector from eq. (4.2), that $S_{[0]}=0$, and for the spatial components

$$
S^{[i]}=\frac{1}{2} \epsilon^{[i j k]} S^{[j k]},
$$

where this actually holds in every locally flat frame, and the indices are just Euclidean here. Therefore, the spin vector is also constant in the body-fixed frame. Hence, the spin-induced higher multipoles are symmetric traceless constant spatial tensors in the body-fixed frame. It should be noted that the constant scalar spin length $S^{2}$, which is the trace of the square of the spin tensor, is absorbed in the mass, and the Wilson coefficients, and is therefore omitted from these traceless tensors.

These even and odd spin-induced multipoles couple to the even and odd parity electric and magnetic curvature tensors, respectively, and their covariant derivatives. The electric and magnetic curvature tensors are usually defined with the Weyl tensor, yet the field here is a vacuum solution at LO, hence Ricci tensor terms can be made to vanish using field redefinitions, and so the use of Weyl and Riemann tensors is equivalent. Then, we define the electric component of the Riemann tensor as

$$
E_{\mu \nu} \equiv R_{\mu \alpha \nu \beta} u^{\alpha} u^{\beta}
$$

and the magnetic component of the Riemann tensor as

$$
B_{\mu \nu} \equiv \frac{1}{2} \epsilon_{\alpha \beta \gamma \mu} R_{\delta \nu}^{\alpha \beta} u^{\gamma} u^{\delta}
$$

where here the dual of the Riemann tensor $* R_{\gamma \mu \delta \nu} \equiv \frac{1}{2} \epsilon_{\alpha \beta \gamma \mu} R_{\delta \nu}^{\alpha \beta}$ is used. In the current work, we consider only couplings linear in Riemann, that is as we noted we are not concerned with the tidal response to external gravitational fields, which does not contribute at the PN orders of interest. 
From the definitions in eqs. (4.10), (4.11), one obtains that both the electric and magnetic components of the Riemann tensor are symmetric, traceless, and orthogonal to the 4-velocity, using the symmetries of the Riemann tensor, the first Bianchi identity, and their being a vacuum field solution. These $\mathrm{SO}(3)$ tensors are then also considered in the body-fixed frame, where only their projection on the spatial triad is non-vanishing due to the covariant gauge of the tetrad. It follows then that they are symmetric and traceless also with respect to their internal spatial indices.

Next, we also consider the covariant derivatives of the electric and magnetic tensors. These are also projected to the body-fixed frame, i.e. $D_{[i]}=e_{[i]}^{\mu} D_{\mu}$, where we clarify that the covariant derivative shall not act on the 4-velocity, contained in $E_{\mu \nu}$ and $B_{\mu \nu}$, since it is a function of the worldline parameter $\sigma$ only. As for the time derivative $D_{[0]}=u^{\mu} D_{\mu} \equiv D / D \sigma$, it is just the covariant derivative along the worldline. Now, at linear order in the curvature time derivatives of the curvature can be integrated by parts to time derivatives of the particle variables. Terms including such higher order time derivatives of the worldline variables can be removed via variable redefinitions with a shift of, e.g. the worldline coordinate, using lower order EOM, and get absorbed into other Riemann dependent finite size operators without higher order time derivatives of the worldline variables, namely into their Wilson coefficients. Therefore, we can consider here only the spatial derivatives, projected orthogonally to $u^{\mu}$.

The indices of the covariant derivatives are also symmetrized among themselves, and with respect to the indices of the electric and magnetic tensors. The first symmetrization follows since the commutation of covariant derivatives leads to further curvature terms, and as only terms linear in the curvature are considered here, such contributions can be taken to vanish. The second symmetrization with indices from the covariant derivatives, and from the electric and magnetic components, follows from the differential Bianchi identity of the Weyl tensor in vacuum, which leads to equations analogous to Maxwell's:

$$
\begin{aligned}
& \epsilon_{[i k l]} D_{[k]} E_{[l j]}=\dot{B}_{[i j]}, \\
& \epsilon_{[i k l]} D_{[k]} B_{[l j]}=-\dot{E}_{[i j]} .
\end{aligned}
$$

Notice that the left hand side contains the commutator of derivative and curvature components indices. Since as was explained time derivatives of the curvature can be ignored at linear order in the curvature, symmetrization follows. Actually, the indices of the covariant derivatives and of the electric and magnetic tensors would anyway be symmetrized here upon contraction with the symmetric spin-induced multipoles. It is also clear from the above discussion, that also in the generic case, where tidal effects are taken into account, the independent curvature tensors are taken as the spatial derivatives of $E_{[i j]}$ and $B_{[i j]}$ with all indices symmetrized, and their time derivatives, as in [49].

Finally, from further contracting from eq. (4.12) we also get

$$
D_{[i]} E_{[i j]}=D_{[i]} B_{[i j]}=0,
$$

analogous to Maxwell's equations, and so we also have similarly

$$
\square E_{[i j]}=\square B_{[i j]}=0 .
$$


Hence, these tensors with covariant derivatives are also traceless. Therefore, the indices of the curvature components, and of their covariant derivatives, should also not be contracted among themselves.

At this stage it becomes clear how to use these building blocks, which we have detailed, to construct the nonminimal couplings with spin in the point particle action. Due to parity invariance the tensors, which contain the even-parity electric or the odd-parity magnetic curvature components, should be contracted with an even or odd number of the spin vector $S^{\mu}$ in eq. (4.2), respectively, of an equal tensor rank. Yet, noting eq. (4.6), one can equivalently use the square of the spin tensor $S^{\alpha}{ }_{\mu} S^{\mu}{ }_{\beta}$ instead of the tensor product of two spin vectors, since these differ by trace and 4 -velocity terms, which vanish in the contraction with the traceless and orthogonal to $u^{\mu}$ curvature tensors.

From reparametrization invariance and the definitions of the electric and magnetic components in eqs. (4.10) and (4.11), we note that all these nonminimal couplings should be divided by the factor $u \equiv \sqrt{u^{2}}$.

Finally, as we noted for the minimal coupling part of the action, and at the end of section 3.2, these nonminimal couplings should be expressed in terms of the rotational gauge variables, using eq. (3.29).

\subsection{Nonminimal couplings to all orders in spin}

Based on the considerations from the previous section, we can actually write down the LO spin-induced nonminimal couplings in eq. (3.3) to all orders in spin in the following:

$$
\begin{aligned}
L_{\mathrm{SI}}= & \sum_{n=1}^{\infty} \frac{(-1)^{n}}{(2 n) !} \frac{C_{E S^{2 n}}}{m^{2 n-1}} D_{\mu_{2 n}} \cdots D_{\mu_{3}} \frac{E_{\mu_{1} \mu_{2}}}{\sqrt{u^{2}}} S^{\mu_{1}} S^{\mu_{2}} \cdots S^{\mu_{2 n-1}} S^{\mu_{2 n}} \\
& +\sum_{n=1}^{\infty} \frac{(-1)^{n}}{(2 n+1) !} \frac{C_{B S^{2 n+1}}}{m^{2 n}} D_{\mu_{2 n+1}} \cdots D_{\mu_{3}} \frac{B_{\mu_{1} \mu_{2}}}{\sqrt{u^{2}}} S^{\mu_{1}} S^{\mu_{2}} \cdots S^{\mu_{2 n-1}} S^{\mu_{2 n}} S^{\mu_{2 n+1}},
\end{aligned}
$$

where in the first term of the first sum it is understood that the covariant derivatives do not exist. The mass exponent in the prefactor is actually set such that the Wilson coefficients are rendered dimensionless. The factorial in the prefactor is fixed from the symmetry of the spin-induced multipole. Also, the sign is alternating for each pair of spins that is added with a corresponding pair of derivatives, which can be seen from considering the definition of the multipole expansion in Fourier space, and then passing to coordinate space. In principle, the numerical factor is fixed such that the Wilson coefficients equal unity for the black hole case. It should be noted that the relation between all Wilson coefficients in eq. (4.16), and the multipole moments used in numerical simulations [50-52], should be worked out using a formal matching procedure. It is clear from [51], that this can present subtleties, and is therefore left for future work. However, the numerical values for the multipoles in [50-52] are expected to lead to good estimates for the Wilson coefficients through an ad hoc identification.

As we already noted these operators are naturally ordered by their PN relevance. For each multipole with $\mathrm{N}$ spins, we have $\mathrm{N}$ derivatives of the field, hence it is suppressed by the ratio $r_{s}^{(2 N-N)} / r^{N}=v^{2 N}$ with respect to the Newtonian point-mass term. Yet, in the 
magnetic odd-parity curvature component, which is coupled to the odd in spin multipoles, the LO coupling must involve the gravito-magnetic vector of the NRG fields (see section 5.1 below, and eq. (5.6) there, to recall the NRG fields), rather than the scalar, which appears as the LO coupling in the even-parity case [28]. At LO the gravito-magnetic vector must be contracted with its LO mass coupling, which already carries one further power of $v$. Therefore, the even multipoles with $2 n$ spins enter at the $(2 n) \mathrm{PN}$ order, whereas the odd multipoles with $(2 n+1)$ spins enter at the $(2 n+1.5) \mathrm{PN}$ order for rapidly rotating compact objects.

Now, we are interested in particular in the spin-induced nonminimal couplings, which contribute up to the $4 \mathrm{PN}$ order for rapidly rotating compact objects, i.e. in the quadrupole, octupole, and hexadecapole. The LO nonminimal coupling of the spin-induced quadrupole, which gives rise to the well-known LO spin-induced finite size effect [53, 54], was noted in [38], though in a different form than what we derive here. The LO nonminimal couplings of the spin-induced octupole and hexadecapole were presented in [28] only recently, where they were also obtained using the EFT formulation for spin, which we introduce here. Let us present then these LO nonminimal couplings explicitly.

LO spin-squared. From eq. (4.16) we can read the LO nonminimal coupling for the spin-induced quadrupole as

$$
L_{E S^{2}}=-\frac{C_{E S^{2}}}{2 m} \frac{E_{\mu \nu}}{\sqrt{u^{2}}} S^{\mu} S^{\nu}
$$

where the quadrupolar deformation constant due to spin from [54], introduced in [38] as the Wilson coefficient $C_{E S^{2}}$, equals unity in the black hole case. For 1.4 solar mass neutron stars a numerical computation yields $C_{E S^{2}} \simeq 4-8$, depending on the equation of state $[50,51]$. This nonminimal coupling contributes as of the $2 \mathrm{PN}$ order for rapidly rotating compact objects.

LO cubic in spin. From eq. (4.16) we read the LO nonminimal coupling for the spininduced octupole as

$$
L_{B S^{3}}=-\frac{C_{B S^{3}}}{6 m^{2}} \frac{D_{\lambda} B_{\mu \nu}}{\sqrt{u^{2}}} S^{\mu} S^{\nu} S^{\lambda}
$$

This nonminimal coupling was introduced in [28], and also recently confirmed in an equivalent form in [55]. The Wilson coefficient $C_{B S^{3}}$ introduced in [28], which encodes the octupolar deformation due to spin, equals unity in the black hole case, where its value for neutron stars was obtained in $[51,52]$. This nonminimal coupling contributes as of the 3.5PN order for rapidly rotating compact objects.

LO quartic in spin. Finally, from eq. (4.16) we also read the LO nonminimal coupling for the spin-induced hexadecapole as

$$
L_{E S^{4}}=\frac{C_{E S^{4}}}{24 m^{3}} \frac{D_{\lambda} D_{\kappa} E_{\mu \nu}}{\sqrt{u^{2}}} S^{\mu} S^{\nu} S^{\lambda} S^{\kappa} .
$$


This nonminimal coupling was also introduced in [28] with the Wilson coefficient $C_{E S^{4}}$, which encodes the hexadecapolar deformation due to spin. Also here this Wilson coefficient equals unity in the black hole case, and its value for neutron stars was obtained in [52]. This nonminimal coupling contributes as of the $4 \mathrm{PN}$ order for rapidly rotating compact objects.

\section{$5 \quad$ Integrating out the orbital scale}

In order to obtain an EFT of radiation, including the conservative sector, we should proceed now to integrate out the field modes below the orbital scale. First, we recall that

$$
\eta_{A B} \hat{e}^{A \mu} \hat{e}^{B \nu}=g^{\mu \nu}
$$

that is the worldline tetrad contains field DOFs, in addition to the worldline rotational DOFs. In order to integrate out the field DOFs, we should disentangle them from the rotational DOFs. For that, we consider a tetrad field, $\tilde{e}_{a \mu}$, such that

$$
\eta_{a b} \tilde{e}^{a}{ }_{\mu} \tilde{e}^{b}{ }_{\nu}=g_{\mu \nu}
$$

and we have that

$$
\hat{e}_{A}^{\mu}=\hat{\Lambda}_{A}^{b} \tilde{e}_{b}^{\mu}
$$

where

$$
\eta^{A B} \hat{\Lambda}_{A}{ }^{a} \hat{\Lambda}_{B}^{b}=\eta^{a b}
$$

Then the rotational DOFs are contained in $\hat{\Lambda}_{A}{ }^{b}$. Notice that $\hat{e}_{A}{ }^{\mu}$ and $\hat{\Lambda}_{A}{ }^{a}$ are only defined on the worldline, whereas $\tilde{e}_{a}{ }^{\mu}$ is a field over spacetime. Actually here $\tilde{e}_{a}{ }^{\mu}$ is the fundamental field, unlike the nonspinning case, which can be formulated only in terms of the metric. An important consequence of this change in the representative DOFs of the field is an additional gauge freedom due to the internal Lorentz invariance of the local tetrad. This will be discussed in section 5.1 below.

Let us then go on to disentangle the field from the worldline DOFs in the action. Then the minimal coupling term from eq. (3.27) can be rewritten as [20]

$$
\frac{1}{2} \hat{S}_{\mu \nu} \hat{\Omega}^{\mu \nu}=\frac{1}{2} \hat{S}_{a b} \hat{\Omega}_{\mathrm{flat}}^{a b}+\frac{1}{2} \hat{S}_{a b} \omega_{\mu}^{a b} u^{\mu}
$$

where we have used the Ricci rotation coefficients, defined in eq. (3.6), $\hat{\Omega}_{\text {flat }}^{a b}=\hat{\Lambda}^{A a} \frac{d \hat{\Lambda}_{A}{ }^{b}}{d \sigma}$ is the locally flat spacetime angular velocity tensor, and $\hat{S}_{a b}=\tilde{e}_{a}^{\mu} \tilde{e}_{b}^{\nu} \hat{S}_{\mu \nu}$ is the spin projected to the local frame. It should be stressed that one should also switch to the spin and angular velocity in the local frame as the fundamental variational variables. Note also that in this form the canonical $\mathrm{SO}(1,3)$ form already emerges, as the first term on the right hand side of eq. (5.5) is the kinematic term, which represents the Poisson brackets, and the Legendre transform in the angular velocity $\hat{\Omega}_{\text {flat }}^{a b}$ is already automatically performed.

Yet the separation of the field from the particle worldline DOFs is not complete. The gauge of the worldline temporal Lorentz matrix, $\hat{\Lambda}_{[0]}{ }^{a}=w^{a}=\tilde{e}^{a}{ }_{\mu} w^{\mu}$, may contain further field dependence, and the temporal components of the local spin also contain in 
general further field dependence [22]. We recall that following [18], in the application of a Routhian approach for spin in $[17,38]$ the SSC are applied at the level of the EOM, similar to traditional methods working at the level of the EOM, see e.g. [56-58]. Therefore, similarly both require further computation of the metric or tetrad field in order to extract the physical EOM [22]. This is in conflict with the definition of the EFT in eq. (2.7), since that means that the field has actually not been completely integrated out, and indeed an EFT, i.e. an effective action at the orbital scale has not been obtained. As was put forward and implemented in [19], the field would be completely disentangled from the worldline DOFs once a gauge for the worldline rotational variables is fixed at the level of the Feynman rules. It should be noted that also in the ADM Hamiltonian formalism the constraints for the spin and for its conjugate DOFs are applied prior to the obtainment of the potential, e.g. in $[59,60]$. The gauge fixing of the rotational variables will be further illustrated in section 5.2 below.

\subsection{Tetrad field gauge}

We recall from eqs. (2.4) and (2.5) that the field modes at the orbital scale are instantaneous at leading order. Therefore, a Kaluza-Klein like reduction over the time dimension at this stage makes a very sensible decomposition of spacetime in the nonrelativistic limit $[25,26]$. This parametrization of the metric, given by

$$
d s^{2}=g_{\mu \nu} d x^{\mu} d x^{\nu} \equiv e^{2 \phi}\left(d t-A_{i} d x^{i}\right)^{2}-e^{-2 \phi} \gamma_{i j} d x^{i} d x^{j}
$$

defines the nonrelativistic gravitational (NRG) fields $\phi, A_{i}$, and $\gamma_{i j} \equiv \delta_{i j}+\sigma_{i j}$. It should be noted that an exponential parametrization of the metric coefficients was already introduced in [61]. Indeed, the NRG fields proved to be advantageous for PN applications both in the non-spinning $[25,26]$, and spinning cases $[19,20,28]$. In addition to providing physical insight and exhibiting a useful hierarchy in the worldline couplings, the NRG fields simplify the propagators, the interaction vertices, and their extraction. We will also see here in section 6.4, that the NRG fields reduce the number of Feynman diagrams in topologies of higher loop order. In addition, the worldline mass couplings are simple and immediate to obtain. We would like to have these benefits for the worldline spin couplings as well, hence we should gauge the tetrad field in a sensible manner adapted to the NRG space+time decomposition of the metric.

The time gauge of Schwinger [62], which is also used with the ADM space+time field decomposition, see e.g. in [60], provides such a sensible gauge for a tetrad of NRG fields. Using the internal local Lorentz invariance of the tetrad field, the time gauge is defined by locking the time axes of the local coordinate systems to the time axis of the general coordinate systems. We take $\tilde{e}_{a}{ }^{0}$ to be a timelike vector in the local frame, and then it is possible to choose each local frame so that the spatial components of $\tilde{e}_{a}{ }^{0}$ vanish. That is, in the time gauge we take

$$
\tilde{e}_{(i)}{ }^{0}(x)=0 .
$$


Then, we have from eqs. (5.2), (5.6), and (5.7), and again using the local Lorentz symmetry, that all the components of the tetrad can be chosen as

$$
\tilde{e}^{a}{ }_{\mu}=\left(\begin{array}{cc}
e^{\phi} & -e^{\phi} A_{i} \\
0 & e^{-\phi} \sqrt{\gamma}_{i j}
\end{array}\right),
$$

where $\sqrt{\gamma}_{i j}$ is the symmetric square root of $\gamma_{i j}$, for which we should solve. Indeed, using the $3+3$ degrees of gauge freedom of the internal Lorentz symmetry, we are left with the 10 DOFs of the metric, out of the 16 DOFs of a general tetrad. Hence, we have a closed form tetrad for the NRG fields apart from $\sqrt{\gamma}_{i j}$, which is conveniently defined, with some trivial components $\tilde{e}_{0}^{a}=e^{\phi}(1, \overrightarrow{0})$.

\subsection{Fixing the gauge of the rotational variables}

In a classical setting one can fix the rotational gauge by simply inserting the rotational constraints in the action before integrating out the field. It should be stressed that if the rotational gauge is not fixed prior to integrating out the field, the orbital field still appears at the level of the EOM, where the SSC should be applied, indicating that the EFT computation is incomplete [22], and see also section 5.4 below.

We recall from eq. (5.5), that we now use the Lorentz matrices, connecting the worldline and local frames, $\hat{\Lambda}_{A}^{a}$, and the spin $\hat{S}_{a b}$ projected to the local frame, as our rotational variables. These still contain gauge freedom, that should be fixed by the gauge conditions applied in the local frame, given in the form

$$
\begin{aligned}
\hat{\Lambda}_{[0] a} & =w_{a}, \\
\hat{S}^{a b}\left(p_{b}+\sqrt{p^{2}} \hat{\Lambda}_{[0] b}\right) & =0 .
\end{aligned}
$$

For similar conditions in the flat spacetime case, see [36]. As we already noted these gauge constraints should be implemented at the level of the action in order to ultimately arrive at an effective action without any remaining orbital scale field modes. We will also see now, that we cannot obtain an effective action formulated with the worldline spin as a further DOF, if we gauge fix the spin without gauge fixing its conjugate DOFs.

We go on now to consider the three sensible options to fix this gauge: the covariant gauge, the canonical gauge, and the gauge of no mass dipole, which we formulate here below. The canonical gauge, which is associated with canonical variables, is of particular interest due to the advantages of such variables for the obtainment of the spin EOM, and of a corresponding Hamiltonian. We will see that in either case, we will ultimately have to switch to the rotational variables in the canonical gauge in order to completely integrate out the field, and get an effective action. Intuitively, this is the consequence of having our point particle action in eq. (3.3), formulated in terms of the spin in addition to its conjugate as independent variables, as we noted for the form of eq. (5.5).

Covariant gauge. We can choose in eq. (5.9)

$$
\hat{\Lambda}_{[0] a}=\frac{p_{a}}{\sqrt{p^{2}}} .
$$


In this case we are back with Tulczyjew's covariant SSC, and the original Lorentz matrices and spin variables, such that it holds for the Lorentz matrices that

$$
\Lambda_{[0]}^{a}=\frac{p^{a}}{\sqrt{p^{2}}} \Leftrightarrow \Lambda_{[i] a} p^{a}=0
$$

Yet, we note that in this case the matrix $\Lambda_{[i]}^{(j)}$ is not an $\mathrm{SO}(3)$ rotation matrix. Instead, from eqs. (5.4) and (5.12), we get that

$$
\Lambda_{[k]}^{(i)} \Lambda^{[k](j)}=\delta^{i j}+\frac{p^{(i)} p^{(j)}}{p^{2}} .
$$

Hence the Lorentz matrices depend on the field through $p^{a}=\tilde{e}^{a}{ }_{\mu} p^{\mu}$. This is not desirable for an EFT approach, as the field DOFs are not separated from the particle DOFs, which would hinder integrating out the field. Moreover, $\Lambda_{[i]}^{(j)}$ is mixing rotational and linear motion DOFs. We can already note that the only way out of this predicament is to redefine $\Lambda_{A}{ }^{a}$ by boosting to the local rest frame. This is what we shall do now, and what is done in the canonical gauge, which we discuss next.

Thus, let us stick here with the covariant gauge for the spin variable. On the upside, we note that the extra term from minimal coupling in eq. (3.31) drops, since the SSC is implemented in the action. Then, let us evaluate $\frac{1}{2} S_{a b} \Omega_{\text {flat }}^{a b}$ from eq. (5.5). Using eq. (3.20) for the locally flat frames, with $w^{a}=\delta_{0}^{a}$ and $q^{a}=p^{a} / \sqrt{p^{2}}$, and implementing the covariant $\mathrm{SSC}$ in the action, we then obtain

$$
\begin{aligned}
\frac{1}{2} S_{a b} \Omega_{\text {flat }}^{a b}= & \frac{1}{2}\left(S_{(i)(j)}-\frac{S_{(i)(k)} p^{(k)} p^{(j)}}{p_{(0)}\left(\sqrt{p^{2}}+p_{(0)}\right)}+\frac{S_{(j)(k)} p^{(k)} p^{(i)}}{p_{(0)}\left(\sqrt{p^{2}}+p_{(0)}\right)}\right) \hat{\Omega}_{\mathrm{flat}}^{(i)(j)} \\
& +\frac{S_{(i)(j)} p^{(j)}}{p_{(0)}\left(\sqrt{p^{2}}+p_{(0)}\right)} \frac{d p^{(i)}}{d \sigma}
\end{aligned}
$$

where the temporal terms of $\Omega_{\text {flat }}^{a b}$ were dropped due to taking $w^{a}=\delta_{0}^{a}$, and

$$
\hat{\Omega}_{\text {flat }}^{(i)(j)}=\hat{\Lambda}^{[k](i)} \frac{d \hat{\Lambda}_{[k]}^{(j)}}{d \sigma} .
$$

This is precisely doing the transformation of the Lorentz matrix in eq. (4.5), using eqs. (4.2), (4.3) from [22], where the matrix $\hat{\Lambda}_{[i]}{ }^{(j)}$ is now an $\mathrm{SO}(3)$ rotation matrix. Indeed, if we also make the spin transformation from eq. (4.6) of [22] in eq. (5.14), we get agreement with eq. (4.7) there. Let us note then that also in eq. (5.14) here, we get an additional term with a derivative of the momentum instead of the extra term from minimal coupling in eq. (3.31), that dropped due to the covariant SSC implemented. It is also already clear that $\hat{\Omega}_{\text {flat }}^{(i)(j)}$ will appear in the spin vertices, and therefore also in the final potential. Therefore, it is clear that the spin variable should indeed be redefined as in [22] in order to simplify the Feynman rules, and to easily obtain the EOM of spin, see section 5.4. This ultimately amounts to going to the canonical gauge. 
Canonical gauge. Let us choose in eq. (5.9) the canonical gauge, that is

$$
\hat{\Lambda}_{[0]}^{a}=\delta_{0}^{a} \Leftrightarrow \hat{\Lambda}_{A}{ }^{(0)}=\delta_{A}^{0} .
$$

Essentially, this means that we have boosted the Lorentz matrices $\Lambda_{A a}$ to the local rest frame, such that they became rotation matrices, decoupled from the linear motion DOFs, and also from the field DOFs. From eqs. (5.16) and (3.22) in the local frame we also get a new spin variable $\hat{S}_{a b}$, which from eqs. (5.16) and (5.10) satisfies the canonical SSC, given in the local frame by

$$
\hat{S}^{a b}\left(p_{b}+\sqrt{p^{2}} \delta_{0 b}\right)=0 .
$$

This is a generalization of the Pryce-Newton-Wigner SSC [63, 64] for curved spacetime. It should be noted that a similar SSC was suggested in eq. (4.7) of [65] for a canonical formalism of a test particle at linear order in the particle spin. Note that their eq. (4.7), formulated in terms of the local tetrad field, is not to be confused with eq. (3.23) here, which is a generic SSC, formulated in terms of the worldline tetrad, where the gauge of the worldline tetrad is not fixed. Further, in [65] they choose the conjugate gauge of the worldline Lorentz matrices as the covariant one. Here the choice of gauge for the worldline tetrad or Lorentz matrices fixes also the spin gauge, namely the corresponding SSC.

Then again similar to eq. (5.14) the temporal components of the Lorentz matrices simply drop, and we get

$$
\frac{1}{2} \hat{S}_{a b} \hat{\Omega}_{\text {flat }}^{a b}=\frac{1}{2} \hat{S}_{(i)(j)} \hat{\Omega}_{\text {flat }}^{(i)(j)} .
$$

This means that we have now the familiar kinematics of a three-dimensional top. The canonical gauge is then useful to disentangle the particle and field DOFs, and is therefore optimal for a formulation of the EFT in terms of the worldline spin, since as discussed for the covariant gauge above, ultimately we have to switch to the rotational variables in the canonical gauge. Hence, we shall fix the spin gauge to the canonical one.

No mass dipole gauge. As a further illustration for a non-covariant spin gauge, we can choose in eq. (5.9)

$$
\hat{\Lambda}_{[0]}^{a}=\frac{2 p_{0} \delta_{0}^{a}-p^{a}}{\sqrt{p^{2}}} .
$$

In this case we are boosting to the local frame, where the mass dipole vanishes, and from eqs. (5.19) and (5.10) we have

$$
\hat{S}_{a 0}=0 .
$$

This is the SSC known from [66].

Shift of the position coordinate. Once a specific non-covariant gauge is fixed for the rotational DOFs, the 'center' of the spinning object, with respect to which the multipoles are considered, will no longer coincide with the spatial origin of the worldline tetrad. Hence one should eventually make a shift in the position coordinate from the worldline coordinate to the position coordinate of the 'center'. This shift of position, would actually be enforced by the reduction of acceleration terms, originating from the extra minimal coupling term in eq. (3.31). It is sensible then to make this shift of position only after the EFT computation 
is through, so that there is no ambiguity in shifting from the worldline coordinate, and in the implementation of the rotational gauge fixing at the level of the point particle action. We shall see in section 6 , that performing the linear shift of position just corresponds to the insertion of lower order EOM for the position. Yet, it should be noted that the quadratic shift of individual positions would contribute to the spin-squared interaction as of the NLO.

As we noted in section 4 the nonminimal couplings in the action are naturally expressed in terms of the projected rotational variables, which we fix to the covariant gauge. Let us then work out the components of the spin, $S_{a b}$, in terms of the spin variable in the canonical gauge. From the canonical SSC in eq. (5.17) we have

$$
\hat{S}_{a(0)}=-\frac{\hat{S}_{a(i)} u^{(i)}}{u+u^{(0)}}=-\hat{S}_{a b} \frac{u^{b}}{u},
$$

and using this in eq. (3.29), we find

$$
\begin{aligned}
S_{(i)(j)} & =\hat{S}_{(i)(j)}-\hat{S}_{(i)(k)} \frac{u_{(j)} u^{(k)}}{u\left(u+u^{(0)}\right)}+\hat{S}_{(j)(k)} \frac{u_{(i)} u^{(k)}}{u\left(u+u^{(0)}\right)}, \\
S_{(i)(0)} & =-\frac{\hat{S}_{(i)(j)} u^{(j)}}{u} .
\end{aligned}
$$

Note that here all velocities are the local tetrad projected ones, not the coordinate velocities. Naturally, one would obtain similar relations upon using the covariant gauge first to eliminate the $S^{0 i}$ components, and then transforming to the canonical gauge variables at the 3-dimensional level as in [22]. Similarly, using eq. (5.16) in eq. (3.30), leads to eq. (4.5) of [22], which we write here as

$$
\Lambda^{[i](j)}=\hat{\Lambda}^{[i](k)}\left(\delta^{(k)(j)}+\frac{u^{(k)} u^{(j)}}{u\left(u+u^{(0)}\right)}\right) .
$$

Let us also work out explicitly the minimal coupling term in terms of the spatial components of the local spin variable in the canonical gauge. From eqs. (3.27), (3.31), and (5.5), we already have

$$
\frac{1}{2} S_{\mu \nu} \Omega^{\mu \nu}=\frac{1}{2} \hat{S}_{a b} \hat{\Omega}_{\text {flat }}^{a b}+\frac{1}{2} \hat{S}_{a b} \omega_{\mu}^{a b} u^{\mu}+\frac{\hat{S}_{a b} u^{b}}{u^{2}} \frac{D u^{a}}{D \sigma},
$$

and note that

$$
\frac{D u^{a}}{D \sigma}=\dot{u}^{a}+\omega_{\mu}{ }^{c a} u_{c} u^{\mu} .
$$

Next we use eqs. (5.18) and (5.21) for the canonical gauge, and get from eqs. (5.25) and (5.26) that

$$
\begin{aligned}
\frac{1}{2} S_{\mu \nu} \Omega^{\mu \nu}= & \frac{1}{2} \hat{S}_{a b} \hat{\Omega}_{\text {flat }}^{a b}-\frac{\hat{S}_{a b} u^{a} \dot{u}^{b}}{u^{2}}+\frac{1}{2} \omega_{\mu}^{a b} u^{\mu}\left(\hat{S}_{a b}-2 \hat{S}_{a c} \frac{u^{c} u_{b}}{u^{2}}\right) \\
= & \frac{1}{2} \hat{S}_{(i)(j)} \hat{\Omega}_{\text {flat }}^{(i)(j)}-\frac{\hat{S}_{(i)(j)} u^{(i)} \dot{u}^{(j)}}{u\left(u+u^{(0)}\right)} \\
& +\frac{1}{2} \omega_{\mu}^{(i)(j)} u^{\mu}\left(\hat{S}_{(i)(j)}-2 \frac{\hat{S}_{(i)(k)} u^{(k)} u_{(j)}}{u\left(u+u^{(0)}\right)}\right)+\omega_{\mu}{ }^{(0)(i)} u^{\mu} \frac{\hat{S}_{(i)(j)} u^{(j)}}{u}
\end{aligned}
$$


Also here for the minimal coupling term it naturally holds, that the same relation is obtained upon first eliminating the $S^{0 i}$ components with the covariant gauge, and then using the 3-dimensional transformations to the canonical gauge variables as in [22].

\subsection{Feynman rules}

From the previous sections 4, 5.1, and 5.2, we can derive all the Feynman rules required up to quadratic level in the spin to NLO. For the nonminimal couplings, which are cubic and quartic in the spin from section 4, and the consequent Feynman rules to LO, we refer to [28], where the cubic and quartic in spin sectors were fully obtained for generic compact binaries. For the Feynman rules, concerning the purely gravitational action in harmonic gauge, and the point-mass nonspinning action worldline couplings from eq. (3.3), we refer to section II of [20]. Similar to [20, 28] we use here the NRG fields [25, 26], and the related tetrad field in the time gauge introduced in section 5.1. Similarly, for the worldline affine parameter we choose the coordinate time $t=y^{0}$, i.e. $\sigma=t$, such that we have $u^{0}=1$, $u^{i}=d y^{i} / d t \equiv v^{i}$. Here we give the Feynman rules for the worldline spin couplings, since only these are modified with respect to previous works, such that they are given here explicitly in terms of the spatial components of the local spin variable in the canonical gauge. Hence from now on we drop the hat notation on the rotational variables, and the round brackets on their indices, and in addition all indices are Euclidean.

From eq. (5.27) we see that we have contributions from kinematic terms involving spin without field coupling. To the order we are considering, these are given by

$$
L_{\mathrm{kin}}=-\vec{S} \cdot \vec{\Omega}-\frac{1}{2}\left(1+\frac{3}{4} v^{2}\right) \epsilon_{i j k} S_{k} v^{j} a^{i}
$$

where $S_{i j}=\epsilon_{i j k} S_{k}, \Omega_{i j}=\epsilon_{i j k} \Omega_{k}$, and $a^{i} \equiv \dot{v}^{i}$. Then, for the one-graviton couplings to the worldline spin the Feynman rules required in this work to NLO are

$$
\begin{aligned}
& \left\{---=\int d t \epsilon_{i j k} S_{k}\left(\frac{1}{2} \partial_{i} A_{j}+\frac{3}{4} v^{i} v^{l}\left(\partial_{l} A_{j}-\partial_{j} A_{l}\right)+v^{i} \partial_{t} A_{j}\right),\right. \\
& \}=\int d t \epsilon_{i j k} S_{k} v^{i}\left(2 \partial_{j} \phi+v^{2} \partial_{j} \phi-2 a^{j} \phi\right), \\
& \hat{\imath}=\int d t \frac{1}{2} \epsilon_{i j k} S_{k} v^{l} \partial_{i} \sigma_{j l}
\end{aligned}
$$

where the oval (gray) blob represents the spin on the worldline. For the two-graviton couplings to the worldline spin, the Feynman rule required here to NLO is:

$$
\delta^{-\cdots}=\int d t 2 \epsilon_{i j k} S_{k} \phi \partial_{i} A_{j} \text {. }
$$


Note that the two-scalar coupling to the worldline spin vanishes in our gauge, which is a desirable feature, reducing the number of total diagrams.

From the $E S^{2}$ nonminimal coupling in eq. (4.17) the Feynman rules for the one-graviton couplings to the worldline spin-squared are given by

$$
\begin{gathered}
\mid=\int d t \frac{C_{E S^{2}}}{2 m}\left[S^{i} S^{j}\left(\partial_{i} \partial_{j} \phi\left(1+\frac{3}{2} v^{2}\right)-3 \partial_{j} \partial_{k} \phi v^{i} v^{k}-2 \partial_{i} \partial_{t} \phi v^{j}\right)\right. \\
\left.-S^{2}\left(\partial_{i} \partial_{i} \phi\left(1+\frac{3}{2} v^{2}\right)-\partial_{i} \partial_{j} \phi v^{i} v^{j}+2 \partial_{i} \partial_{t} \phi v^{i}+2 \partial_{t}^{2} \phi\right)\right], \\
\left.-S^{2}\left(\partial_{i} \partial_{i} A_{k} v^{k}-\partial_{i} \partial_{k} A_{i} v^{k}-\partial_{i} \partial_{t} A_{i}\right)\right]
\end{gathered}
$$

where the square (black) box represents the $E S^{2}$ spin coupling on the worldline. Note that the last terms in the first and second lines of eq. (5.33), and the last four terms on eq. (5.34), are missing from eqs. (40), (39), respectively, in [38]. We should stress that time derivatives of spin should not be dropped before all higher order time derivatives are treated rigorously in the resulting action. Finally, for two-graviton couplings from the worldline $E S^{2}$ term the Feynman rule required for the NLO spin-squared sector is:

$$
\left\lceil=\int d t \frac{C_{E S^{2}}}{2 m}\left[3 S^{i} S^{j}\left(\partial_{i} \phi \partial_{j} \phi+\phi \partial_{i} \partial_{j} \phi\right)-S^{2}\left(\left(\partial_{k} \phi\right)^{2}+3 \phi \partial_{i} \partial_{i} \phi\right)\right] .\right.
$$

\subsection{EOM of the spin}

Using the Feynman rules detailed in the previous section to evaluate the relevant Feynman diagrams, a spin dependent effective action is obtained of the form:

$$
S_{\mathrm{eff}(\text { spin })}=\int d t\left[-\frac{1}{2} \sum_{I=1}^{2} S_{I i j} \Omega_{I}^{i j}-V\left(\vec{x}_{I}, \dot{\vec{x}}_{I}, \ddot{\vec{x}}_{I}, \ldots, S_{I i j}, \dot{S}_{I i j}, \ldots\right)\right],
$$

where here we express the result again in terms of the spin tensor, using $S_{i}=\frac{1}{2} \epsilon_{i j k} S_{j k}$. As explained already in sections 3.1, 5.2 of [22], similar to the EOM of the position, the EOM of the spin should be obtained from a variation of the action, in terms of which the EFT approach is naturally formulated. It should be underlined that one should make an independent variation of this action with respect to the spin, and to its conjugate variables, the rotation matrices. Then the following simple form for the EOM of the spin is obtained

$$
\dot{S}_{I}^{i j}=-4 S_{I}^{k[i} \delta^{j] l} \frac{\delta \int d t V}{\delta S_{I}^{k l}}=-4 S_{I}^{k[i} \delta^{j] l}\left[\frac{\partial V}{\partial S_{I}^{k l}}-\frac{d}{d t} \frac{\partial V}{\partial \dot{S}_{I}^{k l}}+\ldots\right],
$$

see eq. (5.9) in [22].

It should be stressed that the spin EOM in eq. (5.37) are free of the unphysical spin DOFs $S^{0 i}$, and that this is essential in an EFT which removes the orbital scale field. 
However, if one disregards this basic requirement of an EFT, one might also leave the $S^{0 i}$ components of the spin tensor as independent DOFs till after the obtainment of the EOM, as advocated in [17]. In that case the spin dependent action obtained after the evaluation of the relevant Feynman diagrams is of the form [22]:

$$
S_{\text {(spin) }}=\int d t\left[-\frac{1}{2} \sum_{I=1}^{2} S_{I a b} \Omega_{I}^{a b}-V\left(\vec{x}_{I}, \dot{\vec{x}}_{I}, \ddot{\vec{x}}_{I}, \ldots, S_{I a b}, \dot{S}_{I a b}, \ldots\right)\right] .
$$

If we follow the Routhian approach in [17], then we should derive the EOM of the spin in terms of the Poisson brackets of the so $(1,3)$ spin algebra. Since as of NLO we have higher order time derivatives of the spin in the potentials, which actually contribute in the potentials as of NNLO, it is in fact improper to derive the EOM of the spin using Poisson brackets [22].

Again, we can instead make an independent variation of this action with respect to the spin, and to its conjugate Lorentz matrices, to obtain the following EOM of the 4dimensional spin tensor [22]:

$$
\dot{S}_{I}^{a b}=4 S_{I}^{c[a} \eta^{b] d} \frac{\delta \int d t V}{\delta S_{I}^{c d}}=4 S_{I}^{c[a} \eta^{b] d}\left[\frac{\partial V}{\partial S_{I}^{c d}}-\frac{d}{d t} \frac{\partial V}{\partial \dot{S}_{I}^{c d}}+\ldots\right] .
$$

However and more importantly, in that case after the obtainment of the EOM a further EFT computation of the metric at the orbital scale would be required in order to eliminate the $S^{0 i}$ components, and to extract the physical EOM, as was demonstrated in section 3 of [22], since the $S^{0 i}$ components contain orbital scale field DOFs. This situation is actually similar to traditional methods, e.g. in [56-58], where the unconstrained EOM are derived in harmonic coordinates, and then the SSC are inserted, using the metric, which is explicitly computed for the derivation of the unconstrained EOM. This implies that the EFT computation is in fact incomplete when a so(1,3) potential is presented. In the current formulation of an EFT for spin we avoid this undesirable outcome, and directly obtain the physical EOM in the form of eq. (5.37).

\section{Spin potentials to NLO}

In this section we begin to implement the EFT for gravitating spinning objects, which we formulated in the previous sections. We start with the computation of all spin dependent potentials up to quadratic level in the spin to NLO, that is to the $3 \mathrm{PN}$ order for rapidly rotating compact objects. We will use the Feynman rules from section 5.3, with the NRG fields, and the canonical spin gauge. In particular, we also detail here the NLO spin-squared sector, where the NRG fields also turn out to be advantageous, and to remove one-loop topology diagrams, similar to the non-spinning point mass case.

We start by evaluating the Feynman diagrams, as well as the kinematic contributions from eq. (5.28), for each of the sectors. We obtain potentials, which contain only physical DOF, and higher order time derivatives of the variables of the particles, just like the nonspinning potentials. From this point these potentials are handled in the standard manner in 


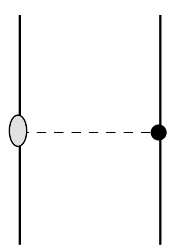

(a)

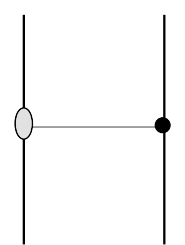

(b)

Figure 1. LO spin-orbit Feynman diagrams. These diagrams should be included together with their mirror images. Note that the value of diagram (a) does not change with respect to [20].

a PN scheme. The EOM of the position, and of the spin, can then be directly obtained via a proper variation of the action, see section 5.4. The higher order time derivatives should be removed at the level of the EOM then, so that the EOM are well-defined. Equivalently, here we first eliminate higher order time derivatives via redefinitions of the position and spin variables at the level of the action, see section 5 in [22]. Again, the EOM of the position and spin can be obtained via a proper variation of the action. Further, the Hamiltonian is obtained by a straightforward Legendre transform.

\subsection{LO spin sectors}

\subsubsection{LO spin-orbit sector}

The LO spin-orbit sector has been worked out in similar terms in section 4 of [20] with the final action in eq. (72) there. We have the same two Feynman diagrams, shown in figure 1, such that from the diagrams we get the total value presented in eq. (71) of [20]. Note that the value of diagram (a) does not depend on the spin gauge, and can be found in eq. (65) of [20]. The value of diagram (b), which depends on the spin gauge, is given by

$$
\text { Figure } 1(\mathrm{~b})=2 \frac{G m_{2}}{r^{2}} \vec{S}_{1} \cdot \vec{v}_{1} \times \vec{n}+1 \leftrightarrow 2,
$$

where $\vec{r} \equiv \vec{y}_{1}-\vec{y}_{2}, r \equiv \sqrt{\vec{r}^{2}}$, and $\vec{n} \equiv \vec{r} / r$. In addition, we have a kinematic contribution in eq. (5.28) from the extra minimal coupling term as noted in eq. (72) of [20], which equals

$$
L_{\mathrm{kin}}=\frac{1}{2} \vec{S}_{1} \cdot \vec{v}_{1} \times \vec{a}_{1}+1 \leftrightarrow 2,
$$

and is acceleration dependent.

The potential and Hamiltonian. All in all, as in eq. (72) of [20], we obtain the potential

$$
V_{\mathrm{SO}}^{\mathrm{LO}}=-2 \frac{G m_{2}}{r^{2}} \vec{S}_{1} \cdot\left[\vec{v}_{1} \times \vec{n}-\vec{v}_{2} \times \vec{n}\right]-\frac{1}{2} \vec{S}_{1} \cdot \vec{v}_{1} \times \vec{a}_{1}+1 \leftrightarrow 2 .
$$

The EOM can be obtained via a variation of the action. We go on to perform a shift of the positions, $\Delta \vec{y}$, according to

$$
\vec{y}_{1} \rightarrow \vec{y}_{1}+\frac{1}{2 m_{1}} \vec{S}_{1} \times \vec{v}_{1}
$$


and similarly for particle 2 with $1 \leftrightarrow 2$, corresponding to the shift in eq. (3.28). The contribution to the action, which is linear in the shifts, removes the acceleration terms, and is equivalent to substituting in the Newtonian EOM of the position. It reads

$$
\Delta V_{\mathrm{SO}}^{\mathrm{LO}}\left(\Delta \vec{y}_{I}\right)=\frac{1}{2} \vec{S}_{1} \cdot \vec{v}_{1} \times \vec{a}_{1}+\frac{G m_{2}}{2 r^{2}} \vec{S}_{1} \cdot \vec{v}_{1} \times \vec{n}+1 \leftrightarrow 2 .
$$

The potential in eqs. (6.3) and (6.5) was first derived in [23]. Then, with a trivial Legendre transform we obtain the Hamiltonian

$$
H_{\mathrm{SO}}^{\mathrm{LO}}=-\frac{G m_{2}}{r^{2}} \vec{S}_{1} \cdot\left[\frac{3}{2} \frac{\vec{p}_{1} \times \vec{n}}{m_{1}}-2 \frac{\vec{p}_{2} \times \vec{n}}{m_{2}}\right]+1 \leftrightarrow 2 .
$$

We note that at this point the EOM can be obtained from the Poisson brackets, where we have now that the position and momentum variables are canonical conjugate to each other, namely

$$
\left\{y_{I}^{i}, p_{J}^{j}\right\}=\delta^{i j} \delta_{I J}
$$

and that the spin variables also satisfy the canonical Poisson bracket relations, namely

$$
\left\{S_{I}^{i}, S_{J}^{j}\right\}=\epsilon^{i j k} S_{I}^{k} \delta_{I J}
$$

Note that further terms linear in each of the shifts in eq. (6.4) contribute to all NLO spin sectors, and correspond to substituting in EOM from the non-spinning 1PN order, and LO SO sectors. They also contribute to the LO cubic in spin sector, corresponding to the insertion of EOM from the LO spin1-spin2 and spin-squared sectors, as noted in [28]. In addition, terms quadratic in each of the shifts contribute to the NLO spin-squared sector. After this shift at LO, one can proceed at NLO to eliminate the remaining higher order time derivatives by insertion of EOM, where one should use the shifted form of the potential.

\subsubsection{LO quadratic in spin sectors}

In the LO spin1-spin2 and spin-squared sectors, which contain a single Feynman diagram each, there is no dependence in the spin gauge, hence there is no change in figure 1 of [19], nor in figure 1 of [28], respectively. The LO spin1-spin2 potential can be found in eq. (11) of [19], and reads

$$
V_{\mathrm{S}_{1} \mathrm{~S}_{2}}^{\mathrm{LO}}=-\frac{G}{r^{3}}\left[\vec{S}_{1} \cdot \vec{S}_{2}-3 \vec{S}_{1} \cdot \vec{n} \vec{S}_{2} \cdot \vec{n}\right]
$$

where $V=-L$, and the LO spin-squared potential can be found in eq. (2.15) of [28], and reads

$$
V_{\mathrm{SS}}^{\mathrm{LO}}=-\frac{C_{1\left(\mathrm{ES}^{2}\right)}}{2} \frac{G m_{2}}{r^{3} m_{1}}\left[S_{1}^{2}-3\left(\vec{S}_{1} \cdot \vec{n}\right)^{2}\right]+1 \leftrightarrow 2 .
$$

The Hamiltonians are identical to the potentials, since they are independent of the velocities. These potentials were first derived in [53]. 


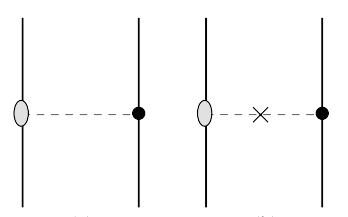

(a)

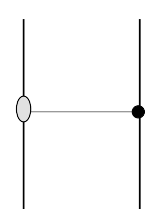

(c)

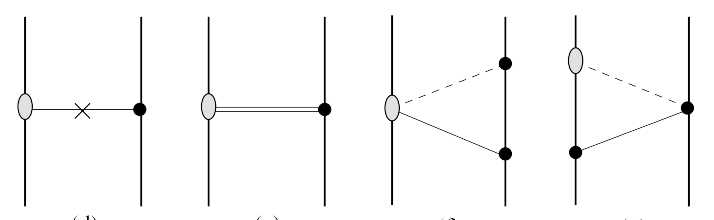

(e)

(f)

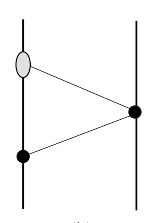

(h)

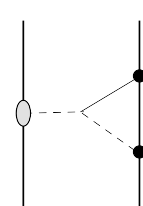

(i)

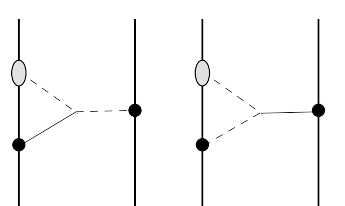

(j)

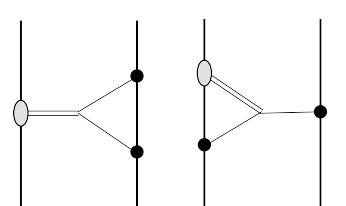

(1)

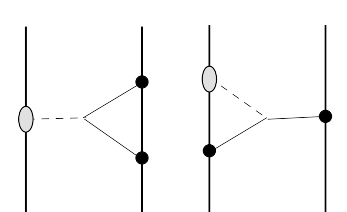

(n)

(o)

Figure 2. NLO spin-orbit Feynman diagrams. These diagrams should be included together with their mirror images. Note that the values of diagrams (b), (e), (g), and (i)-(o) do not change with respect to $[20]$.

\subsection{NLO spin-orbit sector}

Next we compute the NLO spin-orbit sector, for which we essentially have similar Feynman diagrams as detailed in [20], but some changes are found.

First, there is a further kinematic contribution at NLO from eq. (5.28), which equals

$$
L_{\mathrm{kin}}=\frac{3}{8} \vec{S}_{1} \cdot \vec{v}_{1} \times \vec{a}_{1} v_{1}^{2}+1 \leftrightarrow 2 .
$$

Then, we have 15 Feynman diagrams in total in this sector shown in figure 2. Note that the two-scalar spin coupling diagram, which appears as figure 3(b2) in [20], is absent here, since the corresponding spin coupling vanishes in our gauge. The one-loop diagrams, which appear on the bottom row of figure 2, do not change their value with respect to the corresponding figure 4 in [20], since they contain only LO spin couplings, which are independent of the spin gauge. The values of these diagrams can be found in eqs. (101)(107) of [20]. The same is true for diagrams (b), (e), and (g), and their values can be found in eqs. (91), (83), and (94) of [20], respectively. We should note that in eq. (91) of [20] terms containing $\vec{a}_{2} \times \vec{n}, \dot{S}_{i j}$ were already dropped upon the use of LO EOM. Yet, these contribute at NNLO, and moreover all higher order time derivatives should be handled rigorously on the same footing in the resulting action $[22,67]$. Hence, we rewrite here the complete formal result of diagram (b), reading

$$
\begin{aligned}
& \text { Figure 2(b)= } \begin{aligned}
& \frac{G m_{2}}{r^{2}}\left[\vec{S}_{1} \cdot \vec{v}_{1} \times \vec{v}_{2} \vec{v}_{2} \cdot \vec{n}-\vec{S}_{1} \cdot \vec{v}_{2} \times \vec{n} \vec{v}_{1} \cdot \vec{v}_{2}+3 \vec{S}_{1} \cdot \vec{v}_{2} \times \vec{n} \vec{v}_{1} \cdot \vec{n} \vec{v}_{2} \cdot \vec{n}\right] \\
& +\frac{G m_{2}}{r}\left[\vec{S}_{1} \cdot \vec{v}_{1} \times \vec{a}_{2}+\vec{S}_{1} \cdot \vec{a}_{2} \times \vec{n} \vec{v}_{1} \cdot \vec{n}-\dot{\vec{S}}_{1} \cdot \vec{v}_{2} \times \vec{n} \vec{v}_{2} \cdot \vec{n}\right] \\
& -G m_{2} \dot{\vec{S}}_{1} \cdot \vec{a}_{2} \times \vec{n} .
\end{aligned}
\end{aligned}
$$

The following diagrams change value due to the spin gauge, and they are evaluated here as

Figure $2(\mathrm{a})=-\frac{G m_{2}}{r^{2}}\left[3 \vec{S}_{1} \cdot \vec{v}_{1} \times \vec{n} \vec{v}_{1} \cdot \vec{v}_{2}+\vec{S}_{1} \cdot \vec{v}_{2} \times \vec{n} v_{2}^{2}+\vec{S}_{1} \cdot \vec{v}_{1} \times \vec{v}_{2} \vec{v}_{1} \cdot \vec{n}\right]$

$$
+4 \frac{G m_{2}}{r}\left[\vec{S}_{1} \cdot \vec{a}_{1} \times \vec{v}_{2}+\dot{\vec{S}}_{1} \cdot \vec{v}_{1} \times \vec{v}_{2}\right]
$$


Figure $2(\mathrm{c})=\left(v_{1}^{2}+3 v_{2}^{2}\right) \frac{G m_{2}}{r^{2}} \vec{S}_{1} \cdot \vec{v}_{1} \times \vec{n}+2 \frac{G m_{2}}{r} \vec{S}_{1} \cdot \vec{v}_{1} \times \vec{a}_{1}$,

Figure $2(\mathrm{~d})=\frac{G m_{2}}{r^{2}}\left[\vec{S}_{1} \cdot \vec{v}_{1} \times \vec{n} \vec{v}_{1} \cdot \vec{v}_{2}+\vec{S}_{1} \cdot \vec{v}_{1} \times \vec{v}_{2} \vec{v}_{1} \cdot \vec{n}-3 \vec{S}_{1} \cdot \vec{v}_{1} \times \vec{n} \vec{v}_{1} \cdot \vec{n} \vec{v}_{2} \cdot \vec{n}\right]$

$$
+\frac{G m_{2}}{r}\left[\vec{S}_{1} \cdot \vec{v}_{2} \times \vec{a}_{1}+\vec{S}_{1} \cdot \vec{a}_{1} \times \vec{n} \vec{v}_{2} \cdot \vec{n}-\dot{\vec{S}}_{1} \cdot \vec{v}_{1} \times \vec{v}_{2}+\dot{\vec{S}}_{1} \cdot \vec{v}_{1} \times \vec{n} \vec{v}_{2} \cdot \vec{n}\right]
$$

Figure $2(\mathrm{f})=8 \frac{G^{2} m_{2}^{2}}{r^{3}} \vec{S}_{1} \cdot \vec{v}_{2} \times \vec{n}$,

Figure $2(\mathrm{~h})=-2 \frac{G^{2} m_{1} m_{2}}{r^{3}} \vec{S}_{1} \cdot \vec{v}_{1} \times \vec{n}$.

The potential. Summing all contributions from the kinematic term and Feynman diagrams, we obtain the following potential:

$$
\begin{aligned}
& V_{\mathrm{SO}}^{\mathrm{NLO}}=-\frac{G m_{2}}{r^{2}}\left[\vec{S}_{1} \cdot \vec{v}_{1} \times \vec{n} v_{1}^{2}-2 \vec{S}_{1} \cdot \vec{v}_{1} \times \vec{n} \vec{v}_{1} \cdot \vec{v}_{2}+\vec{S}_{1} \cdot \vec{v}_{1} \times \vec{n} v_{2}^{2}\right. \\
& -3 \vec{S}_{1} \cdot \vec{v}_{1} \times \vec{n} \vec{v}_{1} \cdot \vec{n} \vec{v}_{2} \cdot \vec{n}+\vec{S}_{1} \cdot \vec{v}_{2} \times \vec{n} \vec{v}_{1} \cdot \vec{v}_{2}-\vec{S}_{1} \cdot \vec{v}_{2} \times \vec{n} v_{2}^{2} \\
& \left.+3 \vec{S}_{1} \cdot \vec{v}_{2} \times \vec{n} \vec{v}_{1} \cdot \vec{n} \vec{v}_{2} \cdot \vec{n}+\vec{S}_{1} \cdot \vec{v}_{1} \times \vec{v}_{2} \vec{v}_{2} \cdot \vec{n}\right] \\
& +\frac{G^{2} m_{2}^{2}}{2 r^{3}}\left[\vec{S}_{1} \cdot \vec{v}_{1} \times \vec{n}-\vec{S}_{1} \cdot \vec{v}_{2} \times \vec{n}\right]+\frac{3}{8} v_{1}^{2} \vec{S}_{1} \cdot \vec{a}_{1} \times \vec{v}_{1} \\
& +\frac{G m_{2}}{r}\left[2 \vec{S}_{1} \cdot \vec{a}_{1} \times \vec{v}_{1}-3 \vec{S}_{1} \cdot \vec{a}_{1} \times \vec{v}_{2}+\vec{S}_{1} \cdot \vec{a}_{2} \times \vec{v}_{1}-\vec{S}_{1} \cdot \vec{a}_{1} \times \vec{n} \vec{v}_{2} \cdot \vec{n}\right. \\
& \left.-\vec{S}_{1} \cdot \vec{a}_{2} \times \vec{n} \vec{v}_{1} \cdot \vec{n}-\dot{\vec{S}}_{1} \cdot \vec{v}_{1} \times \vec{n} \vec{v}_{2} \cdot \vec{n}+\dot{\vec{S}}_{1} \cdot \vec{v}_{2} \times \vec{n} \vec{v}_{2} \cdot \vec{n}-3 \dot{\vec{S}}_{1} \cdot \vec{v}_{1} \times \vec{v}_{2}\right] \\
& +G m_{2} \dot{\vec{S}}_{1} \cdot \vec{a}_{2} \times \vec{n}+1 \leftrightarrow 2 .
\end{aligned}
$$

As we noted in the LO spin-orbit sector, we go on to perform a shift of the positions according to eq. (6.4), and get contributions linear in the shift from the 1PN order potential, corresponding to the insertion of $1 \mathrm{PN}$ order EOM. This contribution reads

$$
\begin{aligned}
\Delta V_{\mathrm{SO}}^{\mathrm{NLO}}\left(\Delta \vec{y}_{I}\right)= & \frac{1}{4} \vec{S}_{1} \cdot \vec{v}_{1} \times \vec{a}_{1} v_{1}^{2} \\
& +\frac{G m_{2}}{r}\left[\frac{3}{2} \vec{S}_{1} \cdot \vec{v}_{1} \times \vec{a}_{1}-\frac{7}{4} \vec{S}_{1} \cdot \vec{v}_{1} \times \vec{a}_{2}-\frac{1}{4} \vec{S}_{1} \cdot \vec{v}_{1} \times \vec{n} \vec{a}_{2} \cdot \vec{n}\right] \\
& +\frac{G m_{2}}{r^{2}}\left[\frac{3}{4} \vec{S}_{1} \cdot \vec{v}_{1} \times \vec{n} v_{1}^{2}-2 \vec{S}_{1} \cdot \vec{v}_{1} \times \vec{n} \vec{v}_{1} \cdot \vec{v}_{2}+\vec{S}_{1} \cdot \vec{v}_{1} \times \vec{n} v_{2}^{2}\right. \\
& \left.+2 \vec{S}_{1} \cdot \vec{v}_{1} \times \vec{v}_{2} \vec{v}_{1} \cdot \vec{n}-\frac{3}{2} \vec{S}_{1} \cdot \vec{v}_{1} \times \vec{v}_{2} \vec{v}_{2} \cdot \vec{n}-\frac{3}{4} \vec{S}_{1} \cdot \vec{v}_{1} \times \vec{n}\left(\vec{v}_{2} \cdot \vec{n}\right)^{2}\right] \\
& -\frac{G^{2} m_{2}\left(m_{1}+m_{2}\right)}{2 r^{3}} \vec{S}_{1} \cdot \vec{v}_{1} \times \vec{n}+1 \leftrightarrow 2 .
\end{aligned}
$$

After the shift from LO, we proceed to eliminate the remaining higher order time derivatives by insertion of EOM, where we use the shifted form of the potential. For completeness we present the explicit NLO redefinition of position, which removes the higher order time 
derivatives of position in the potential. It reads

$$
\begin{aligned}
& \vec{y}_{1} \rightarrow \vec{y}_{1}+\frac{1}{8 m_{1}} \vec{S}_{1} \times \vec{v}_{1} v_{1}^{2}+\frac{G m_{2}}{m_{1} r}\left(\frac{1}{2} \vec{S}_{1} \times \vec{v}_{1}-3 \vec{S}_{1} \times \vec{v}_{2}-\vec{S}_{1} \times \vec{n} \vec{v}_{2} \cdot \vec{n}\right) \\
&+\frac{G}{r}\left(\frac{11}{4} \vec{S}_{2} \times \vec{v}_{2}-\vec{S}_{2} \times \vec{n} \vec{v}_{2} \cdot \vec{n}+\frac{1}{4} \vec{n} \vec{S}_{2} \cdot \vec{v}_{2} \times \vec{n}\right),
\end{aligned}
$$

and similarly for particle 2 with $1 \leftrightarrow 2$. The contribution to the action, which is linear in this shift, removes the acceleration terms, and is equivalent to substituting in the EOM of the position.

Finally, we note that at NLO spin-orbit there also appear higher order time derivatives of spin, of which a generic rigorous treatment was shown in [22], see section 5 there. According to this treatment, and considering the relevant terms in eq. (6.18), we realize that whereas the position shift of eq. (6.4) was formally a 1PN order shift of the position, here a $2 \mathrm{PN}$ order redefinition of the spin is required. Terms quadratic in the spin shift thus contribute only at the next-to-NNLO (NNNLO) level. Therefore, we can consider only the linear in spin shift, which amounts to the insertion of the EOM of the spin. Here only the LO Newtonian EOM of the spin contribute, that is $\dot{S}_{i j}=0$. Again, for completeness we present the explicit redefinition of spin, which removes its higher order time derivatives in the potential. We use the notation from [22] for the antisymmetric generator of rotation $\omega_{1}^{i j}$, which transforms the spin variable, and reads

$$
\omega_{1}^{i j}=\frac{G m_{2}}{r}\left(3 v_{1}^{i} v_{2}^{j}+v_{1}^{i} n^{j} \vec{v}_{2} \cdot \vec{n}-v_{2}^{i} n^{j} \vec{v}_{2} \cdot \vec{n}-(i \leftrightarrow j)\right),
$$

and similarly for particle 2 with $1 \leftrightarrow 2$. The contribution to the action, linear in this shift, removes the precession terms, and is equivalent to substituting in the EOM of the spin.

The Hamiltonian. At this stage one can perform a straightforward Legendre transform, see e.g. section 6 in [22]. Then, we obtain the following Hamiltonian:

$$
\begin{aligned}
H_{\mathrm{SO}}^{\mathrm{NLO}}=\frac{G m_{2}}{r^{2}}\left[\frac{5}{8} \vec{S}_{1} \cdot \frac{\vec{p}_{1} \times \vec{n}}{m_{1}} \frac{p_{1}^{2}}{m_{1}^{2}}+3 \vec{S}_{1} \cdot \frac{\vec{p}_{1} \times \vec{n}}{m_{1}} \frac{\vec{p}_{1} \cdot \vec{n}}{m_{1}} \frac{\vec{p}_{2} \cdot \vec{n}}{m_{2}}-\frac{3}{4} \vec{S}_{1} \cdot \frac{\vec{p}_{1} \times \vec{n}}{m_{1}}\left(\frac{\overrightarrow{p_{2}} \cdot \vec{n}}{m_{2}}\right)^{2}\right. \\
\quad-\vec{S}_{1} \cdot \frac{\overrightarrow{p_{2}} \times \vec{n}}{m_{2}} \frac{\overrightarrow{p_{1}} \cdot \vec{p}_{2}}{m_{1} m_{2}}-3 \vec{S}_{1} \cdot \frac{\vec{p}_{2} \times \vec{n}}{m_{2}} \frac{\overrightarrow{p_{1}} \cdot \vec{n}}{m_{1}} \frac{\overrightarrow{p_{2}} \cdot \vec{n}}{m_{2}} \\
\left.+2 \vec{S}_{1} \cdot \frac{\vec{p}_{1} \times \vec{p}_{2}}{m_{1} m_{2}} \frac{\vec{p}_{1} \cdot \vec{n}}{m_{1}}-\frac{5}{2} \vec{S}_{1} \cdot \frac{\vec{p}_{1} \times \vec{p}_{2}}{m_{1} m_{2}} \frac{\vec{p}_{2} \cdot \vec{n}}{m_{2}}\right] \\
+\frac{G^{2} m_{1} m_{2}}{r^{3}}\left[\frac{7}{2} \vec{S}_{1} \cdot \frac{\vec{p}_{1} \times \vec{n}}{m_{1}}-6 \vec{S}_{1} \cdot \frac{\vec{p}_{2} \times \vec{n}}{m_{2}}\right] \\
+\frac{G^{2} m_{2}^{2}}{r^{3}}\left[5 \vec{S}_{1} \cdot \frac{\vec{p}_{1} \times \vec{n}}{m_{1}}-\frac{35}{4} \vec{S}_{1} \cdot \frac{\vec{p}_{2} \times \vec{n}}{m_{2}}\right]+1 \leftrightarrow 2 .
\end{aligned}
$$

To verify equivalence with the ADM Hamiltonian result, which was first obtained in [59], after the corresponding EOM were obtained in [56, 68], we resolve the difference between the result in [59], and the result here, using canonical transformations, see e.g. section 7 in [22]. Using the generator of canonical transformations given in eq. (7.8) there with the 


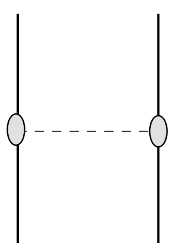

(a)

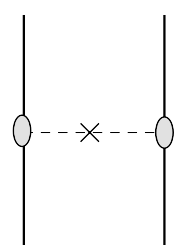

(b)

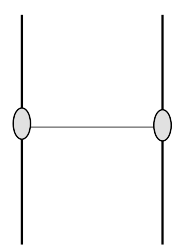

(c)

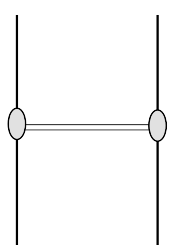

(d)

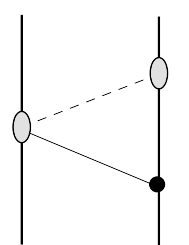

(e)

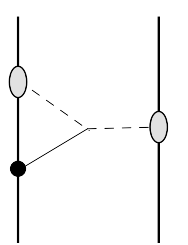

(f)

Figure 3. NLO spin1-spin2 Feynman diagrams. Diagrams (e) and (f) should be included together with their mirror images. Note that the values of diagrams (b), (d), and (f) do not change with respect to [19].

same notations, we find that the difference is resolved with the coefficients of the generator being set to

$$
g_{1}=\frac{5}{4}, \quad g_{3}=\frac{3}{4},
$$

and the remaining coefficients set to zero, hence indeed the equivalence is established.

\subsection{NLO spin1-spin2 sector}

We go on to handle the NLO spin1-spin2 sector, for which we have similar Feynman diagrams as in [19], but again some of the values of the diagrams are modified.

There are 6 Feynman diagrams in this sector shown in figure 3. Again, the values of diagrams (b), (d) and (f), in figure 3 do not change with respect to [19], since they contain only LO spin couplings, which are independent of the spin gauge. Their values can be found in eqs. (25), (14), and (32), respectively, of [19]. Again, in eq. (25) of [19] terms with time derivatives of spin were already dropped upon the use of LO EOM, and hence we rewrite here the complete formal result of diagram (b), given by

Figure 3(b) $=-\frac{G}{2 r^{3}}\left[\vec{S}_{1} \cdot \vec{S}_{2} \vec{v}_{1} \cdot \vec{v}_{2}-\vec{S}_{1} \cdot \vec{v}_{1} \vec{S}_{2} \cdot \vec{v}_{2}-\vec{S}_{1} \cdot \vec{v}_{2} \vec{S}_{2} \cdot \vec{v}_{1}-3 \vec{S}_{1} \cdot \vec{S}_{2} \vec{v}_{1} \cdot \vec{n} \vec{v}_{2} \cdot \vec{n}\right.$

$$
\begin{aligned}
&+3 \vec{S}_{1} \cdot \vec{v}_{1} \vec{S}_{2} \cdot \vec{n} \vec{v}_{2} \cdot \vec{n}+3 \vec{S}_{1} \cdot \vec{v}_{2} \vec{S}_{2} \cdot \vec{n} \vec{v}_{1} \cdot \vec{n}+3 \vec{S}_{1} \cdot \vec{n} \vec{S}_{2} \cdot \vec{v}_{1} \vec{v}_{2} \cdot \vec{n} \\
&\left.+3 \vec{S}_{1} \cdot \vec{n} \vec{S}_{2} \cdot \vec{v}_{2} \vec{v}_{1} \cdot \vec{n}+3 \vec{S}_{1} \cdot \vec{n} \vec{S}_{2} \cdot \vec{n} \vec{v}_{1} \cdot \vec{v}_{2}-15 \vec{S}_{1} \cdot \vec{n} \vec{S}_{2} \cdot \vec{n} \vec{v}_{1} \cdot \vec{n} \vec{v}_{2} \cdot \vec{n}\right] \\
&-\frac{G}{2 r^{2}}\left[\dot{\vec{S}}_{1} \cdot \vec{S}_{2} \vec{v}_{2} \cdot \vec{n}-\dot{\vec{S}}_{1} \cdot \vec{v}_{2} \vec{S}_{2} \cdot \vec{n}-\dot{\vec{S}}_{1} \cdot \vec{n} \vec{S}_{2} \cdot \vec{v}_{2}+3 \dot{\vec{S}}_{1} \cdot \vec{n} \vec{S}_{2} \cdot \vec{n} \vec{v}_{2} \cdot \vec{n}\right] \\
&+1 \leftrightarrow 2-\frac{G}{2 r}\left[\dot{\vec{S}}_{1} \cdot \dot{\vec{S}}_{2}+\dot{\vec{S}}_{1} \cdot \vec{n} \dot{\vec{S}}_{2} \cdot \vec{n}\right] .
\end{aligned}
$$

The values of the following diagrams are modified due to the spin gauge, and now read

$$
\begin{aligned}
& \text { Figure } 3(\mathrm{a})=\frac{G}{2 r^{3}}\left[7 \vec{S}_{1} \cdot \vec{S}_{2} v_{1}^{2}-7 \vec{S}_{1} \cdot \vec{v}_{1} \vec{S}_{2} \cdot \vec{v}_{1}-12 \vec{S}_{1} \cdot \vec{S}_{2}\left(\vec{v}_{1} \cdot \vec{n}\right)^{2}+9 \vec{S}_{1} \cdot \vec{v}_{1} \vec{S}_{2} \cdot \vec{n} \vec{v}_{1} \cdot \vec{n}\right. \\
& \left.\quad+12 \vec{S}_{1} \cdot \vec{n} \vec{S}_{2} \cdot \vec{v}_{1} \vec{v}_{1} \cdot \vec{n}-9 \vec{S}_{1} \cdot \vec{n} \vec{S}_{2} \cdot \vec{n} v_{1}^{2}\right]+1 \leftrightarrow 2 \\
& \\
& \quad+2 \frac{G}{r^{2}}\left[\dot{\vec{S}}_{1} \cdot \vec{S}_{2} \vec{v}_{1} \cdot \vec{n}-\dot{\vec{S}}_{1} \cdot \vec{n} \vec{S}_{2} \cdot \vec{v}_{1}+\vec{S}_{1} \cdot \vec{S}_{2} \vec{a}_{1} \cdot \vec{n}-\vec{S}_{1} \cdot \vec{n} \vec{S}_{2} \cdot \vec{a}_{1}\right]+1 \leftrightarrow 2,
\end{aligned}
$$


Figure $3(\mathrm{c})=-8 \frac{G}{r^{3}}\left[\vec{S}_{1} \cdot \vec{S}_{2} \vec{v}_{1} \cdot \vec{v}_{2}-\vec{S}_{1} \cdot \vec{v}_{2} \vec{S}_{2} \cdot \vec{v}_{1}-\frac{3}{2}\left(\vec{S}_{1} \cdot \vec{S}_{2} \vec{v}_{1} \cdot \vec{n} \vec{v}_{2} \cdot \vec{n}\right.\right.$

$$
\left.\left.-\vec{S}_{1} \cdot \vec{v}_{2} \vec{S}_{2} \cdot \vec{n} \vec{v}_{1} \cdot \vec{n}-\vec{S}_{1} \cdot \vec{n} \vec{S}_{2} \cdot \vec{v}_{1} \vec{v}_{2} \cdot \vec{n}+\vec{S}_{1} \cdot \vec{n} \vec{S}_{2} \cdot \vec{n} \vec{v}_{1} \cdot \vec{v}_{2}\right)\right]
$$

Figure $3(\mathrm{e})=-4 \frac{G^{2}\left(m_{1}+m_{2}\right)}{r^{4}}\left[\vec{S}_{1} \cdot \vec{S}_{2}-3 \vec{S}_{1} \cdot \vec{n} \vec{S}_{2} \cdot \vec{n}\right]$.

We note that in diagrams (c) and (d) double scalar triple products were transformed to scalar products according to the appropriate identity, e.g. in eq. (6.11) of [22].

The potential. Summing all Feynman diagrams, we obtain the following potential:

$$
\begin{aligned}
& V_{\mathrm{S}_{1} \mathrm{~S}_{2}}^{\mathrm{NLO}}=-\frac{G}{r^{3}}\left[\frac{7}{2} \vec{S}_{1} \cdot \vec{S}_{2} v_{1}^{2}-\frac{15}{2} \vec{S}_{1} \cdot \vec{S}_{2} \vec{v}_{1} \cdot \vec{v}_{2}+\frac{7}{2} \vec{S}_{1} \cdot \vec{S}_{2} v_{2}^{2}-\frac{7}{2} \vec{S}_{1} \cdot \vec{v}_{1} \vec{S}_{2} \cdot \vec{v}_{1}\right. \\
& +\frac{5}{2} \vec{S}_{1} \cdot \vec{v}_{1} \vec{S}_{2} \cdot \vec{v}_{2}+\frac{9}{2} \vec{S}_{1} \cdot \vec{v}_{2} \vec{S}_{2} \cdot \vec{v}_{1}-\frac{7}{2} \vec{S}_{1} \cdot \vec{v}_{2} \vec{S}_{2} \cdot \vec{v}_{2}-6 \vec{S}_{1} \cdot \vec{S}_{2}\left(\overrightarrow{v_{1}} \cdot \vec{n}\right)^{2} \\
& +\frac{21}{2} \vec{S}_{1} \cdot \vec{S}_{2} \vec{v}_{1} \cdot \vec{n} \vec{v}_{2} \cdot \vec{n}-6 \vec{S}_{1} \cdot \vec{S}_{2}\left(\vec{v}_{2} \cdot \vec{n}\right)^{2}+\frac{9}{2} \vec{S}_{1} \cdot \vec{v}_{1} \vec{S}_{2} \cdot \vec{n} \vec{v}_{1} \cdot \vec{n} \\
& -\frac{9}{2} \vec{S}_{1} \cdot \vec{v}_{1} \vec{S}_{2} \cdot \vec{n} \vec{v}_{2} \cdot \vec{n}-\frac{15}{2} \vec{S}_{1} \cdot \vec{v}_{2} \vec{S}_{2} \cdot \vec{n} \vec{v}_{1} \cdot \vec{n}+6 \vec{S}_{1} \cdot \vec{v}_{2} \vec{S}_{2} \cdot \vec{n} \vec{v}_{2} \cdot \vec{n} \\
& +6 \vec{S}_{1} \cdot \vec{n} \vec{S}_{2} \cdot \vec{v}_{1} \vec{v}_{1} \cdot \vec{n}-\frac{15}{2} \vec{S}_{1} \cdot \vec{n} \vec{S}_{2} \cdot \vec{v}_{1} \vec{v}_{2} \cdot \vec{n}-\frac{9}{2} \vec{S}_{1} \cdot \vec{n} \vec{S}_{2} \cdot \vec{v}_{2} \vec{v}_{1} \cdot \vec{n} \\
& +\frac{9}{2} \vec{S}_{1} \cdot \vec{n} \vec{S}_{2} \cdot \vec{v}_{2} \vec{v}_{2} \cdot \vec{n}-\frac{9}{2} \vec{S}_{1} \cdot \vec{n} \vec{S}_{2} \cdot \vec{n} v_{1}^{2}+\frac{21}{2} \vec{S}_{1} \cdot \vec{n} \vec{S}_{2} \cdot \vec{n} \vec{v}_{1} \cdot \vec{v}_{2} \\
& \left.-\frac{9}{2} \vec{S}_{1} \cdot \vec{n} \vec{S}_{2} \cdot \vec{n} v_{2}^{2}+\frac{15}{2} \vec{S}_{1} \cdot \vec{n} \vec{S}_{2} \cdot \vec{n} \vec{v}_{1} \cdot \vec{n} \vec{v}_{2} \cdot \vec{n}\right] \\
& +2 \frac{G^{2}\left(m_{1}+m_{2}\right)}{r^{4}}\left[\vec{S}_{1} \cdot \vec{S}_{2}-4 \vec{S}_{1} \cdot \vec{n} \vec{S}_{2} \cdot \vec{n}\right] \\
& -\frac{G}{r^{2}}\left[2 \vec{S}_{1} \cdot \vec{S}_{2} \vec{a}_{1} \cdot \vec{n}-2 \vec{S}_{1} \cdot \vec{S}_{2} \vec{a}_{2} \cdot \vec{n}+2 \vec{S}_{1} \cdot \vec{a}_{2} \vec{S}_{2} \cdot \vec{n}-2 \vec{S}_{1} \cdot \vec{n} \vec{S}_{2} \cdot \vec{a}_{1}\right. \\
& +2 \dot{\vec{S}}_{1} \cdot \vec{S}_{2} \vec{v}_{1} \cdot \vec{n}-\frac{1}{2} \dot{\vec{S}}_{1} \cdot \vec{S}_{2} \vec{v}_{2} \cdot \vec{n}+\frac{1}{2} \vec{S}_{1} \cdot \dot{\vec{S}}_{2} \vec{v}_{1} \cdot \vec{n}-2 \vec{S}_{1} \cdot \dot{\vec{S}}_{2} \vec{v}_{2} \cdot \vec{n} \\
& +\frac{1}{2} \dot{\vec{S}}_{1} \cdot \vec{v}_{2} \vec{S}_{2} \cdot \vec{n}-\frac{1}{2} \vec{S}_{1} \cdot \vec{n} \dot{\vec{S}}_{2} \cdot \vec{v}_{1}-2 \dot{\vec{S}}_{1} \cdot \vec{n} \vec{S}_{2} \cdot \vec{v}_{1}+\frac{1}{2} \dot{\vec{S}}_{1} \cdot \vec{n} \vec{S}_{2} \cdot \vec{v}_{2} \\
& \left.-\frac{1}{2} \vec{S}_{1} \cdot \vec{v}_{1} \dot{\vec{S}}_{2} \cdot \vec{n}+2 \vec{S}_{1} \cdot \vec{v}_{2} \dot{\vec{S}}_{2} \cdot \vec{n}-\frac{3}{2} \dot{\vec{S}}_{1} \cdot \vec{n} \vec{S}_{2} \cdot \vec{n} \vec{v}_{2} \cdot \vec{n}+\frac{3}{2} \vec{S}_{1} \cdot \vec{n} \dot{\vec{S}}_{2} \cdot \vec{n} \vec{v}_{1} \cdot \vec{n}\right] \\
& +\frac{G}{2 r}\left[\dot{\vec{S}}_{1} \cdot \dot{\vec{S}}_{2}+\dot{\vec{S}}_{1} \cdot \vec{n} \dot{\vec{S}}_{2} \cdot \vec{n}\right] .
\end{aligned}
$$

As noted, we go on to perform a shift of the positions according to eq. (6.4), and get contributions linear in each of the shifts from the Newtonian and LO spin-orbit potentials, corresponding to the insertion of the EOM from these sectors. This contribution reads

$$
\begin{aligned}
\Delta V_{\mathrm{S}_{1} \mathrm{~S}_{2}}^{\mathrm{NLO}}\left(\Delta \overrightarrow{y_{I}}\right)=\frac{G}{r^{3}}[ & \vec{S}_{1} \cdot \vec{S}_{2} v_{1}^{2}-\frac{3}{2} \vec{S}_{1} \cdot \vec{S}_{2} \vec{v}_{1} \cdot \vec{v}_{2}+\vec{S}_{1} \cdot \vec{S}_{2} v_{2}^{2}-\vec{S}_{1} \cdot \vec{v}_{1} \vec{S}_{2} \cdot \vec{v}_{1} \\
& +\frac{3}{2} \vec{S}_{1} \cdot \vec{v}_{2} \vec{S}_{2} \cdot \vec{v}_{1}-\vec{S}_{1} \cdot \vec{v}_{2} \vec{S}_{2} \cdot \vec{v}_{2}-\frac{3}{4} \vec{S}_{1} \cdot \vec{S}_{2} \vec{v}_{1} \cdot \vec{n} \vec{v}_{2} \cdot \vec{n}
\end{aligned}
$$




$$
\begin{aligned}
& +3 \vec{S}_{1} \cdot \vec{v}_{1} \vec{S}_{2} \cdot \vec{n} \vec{v}_{1} \cdot \vec{n}-\frac{9}{4} \vec{S}_{1} \cdot \vec{v}_{2} \vec{S}_{2} \cdot \vec{n} \vec{v}_{1} \cdot \vec{n}-\frac{9}{4} \vec{S}_{1} \cdot \vec{n} \vec{S}_{2} \cdot \vec{v}_{1} \vec{v}_{2} \cdot \vec{n} \\
& +3 \vec{S}_{1} \cdot \vec{n} \vec{S}_{2} \cdot \vec{v}_{2} \vec{v}_{2} \cdot \vec{n}-3 \vec{S}_{1} \cdot \vec{n} \vec{S}_{2} \cdot \vec{n} v_{1}^{2}+\frac{21}{4} \vec{S}_{1} \cdot \vec{n} \vec{S}_{2} \cdot \vec{n} \vec{v}_{1} \cdot \vec{v}_{2} \\
& \left.-3 \vec{S}_{1} \cdot \vec{n} \vec{S}_{2} \cdot \vec{n} v_{2}^{2}\right] .
\end{aligned}
$$

After the LO shift, we proceed to eliminate the remaining higher order time derivatives, including the time derivatives of spin, which appear here too, by insertion of EOM, where one should use the shifted form of the potential. For completeness we present the explicit NLO redefinition of position, which removes its higher order time derivatives in the potential. It reads

$$
\vec{y}_{1} \rightarrow \vec{y}_{1}+2 \frac{G}{m_{1} r^{2}}\left(\vec{n} \vec{S}_{1} \cdot \vec{S}_{2}-\vec{S}_{2} \vec{S}_{1} \cdot \vec{n}\right),
$$

and similarly for particle 2 with $1 \leftrightarrow 2$. The contribution to the action, linear in this shift, removes the acceleration terms, and is equivalent to substituting in the EOM of the position.

Here again only the LO Newtonian EOM of spin contribute. Again, for completeness we present the explicit redefinition of spin, which removes its higher order time derivatives, using the antisymmetric generator of rotation $\omega_{1}^{i j}$, which reads

$$
\omega_{1}^{i j}=\frac{G}{r^{2}}\left(\left(2 S_{2}^{i k} v_{1}^{j} n^{k}-\frac{1}{2} S_{2}^{i k} v_{2}^{j} n^{k}-\frac{3}{2} S_{2}^{i k} n^{j} v_{2}^{k}+\frac{3}{2} S_{2}^{i k} n^{j} n^{k} \vec{v}_{2} \cdot \vec{n}\right)-(i \leftrightarrow j)-S_{2}^{i j} \vec{v}_{2} \cdot \vec{n}\right),
$$

and similarly for particle 2 with $1 \leftrightarrow 2$. The contribution to the action, linear in this shift, removes the precession terms, and is equivalent to substituting in the EOM of the spin.

The Hamiltonian. Again, at this stage one can perform a straightforward Legendre transform to obtain the following Hamiltonian:

$$
\begin{aligned}
H_{\mathrm{S}_{1} \mathrm{~S}_{2}}^{\mathrm{NLO}}=-\frac{G}{r^{3}} & {\left[\frac{5}{2} \vec{S}_{1} \cdot \vec{S}_{2} \frac{p_{1}^{2}}{m_{1}^{2}}-6 \vec{S}_{1} \cdot \vec{S}_{2} \frac{\vec{p}_{1} \cdot \vec{p}_{2}}{m_{1} m_{2}}+\frac{5}{2} \vec{S}_{1} \cdot \vec{S}_{2} \frac{p_{2}^{2}}{m_{2}^{2}}-\frac{5}{2} \frac{\vec{S}_{1} \cdot \vec{p}_{1}}{m_{1}} \frac{\vec{S}_{2} \cdot \vec{p}_{1}}{m_{1}}\right.} \\
& +\frac{5}{2} \frac{\vec{S}_{1} \cdot \vec{p}_{1}}{m_{1}} \frac{\vec{S}_{2} \cdot \vec{p}_{2}}{m_{2}}+3 \frac{\vec{S}_{1} \cdot \vec{p}_{2}}{m_{2}} \frac{\vec{S}_{2} \cdot \vec{p}_{1}}{m_{1}}-\frac{5}{2} \frac{\vec{S}_{1} \cdot \vec{p}_{2}}{m_{2}} \frac{\vec{S}_{2} \cdot \vec{p}_{2}}{m_{2}}-6 \vec{S}_{1} \cdot \vec{S}_{2}\left(\frac{\vec{p}_{1} \cdot \vec{n}}{m_{1}}\right)^{2} \\
& +\frac{45}{4} \vec{S}_{1} \cdot \vec{S}_{2} \frac{\vec{p}_{1} \cdot \vec{n}}{m_{1}} \frac{\vec{p}_{2} \cdot \vec{n}}{m_{2}}-6 \vec{S}_{1} \cdot \vec{S}_{2}\left(\frac{\vec{p}_{2} \cdot \vec{n}}{m_{2}}\right)^{2}+\frac{3}{2} \frac{\vec{S}_{1} \cdot \vec{p}_{1}}{m_{1}} \vec{S}_{2} \cdot \vec{n} \frac{\vec{p}_{1} \cdot \vec{n}}{m_{1}} \\
& -\frac{9}{2} \frac{\vec{S}_{1} \cdot \vec{p}_{1}}{m_{1}} \vec{S}_{2} \cdot \vec{n} \frac{\vec{p}_{2} \cdot \vec{n}}{m_{2}}-\frac{21}{4} \frac{\vec{S}_{1} \cdot \vec{p}_{2}}{m_{2}} \vec{S}_{2} \cdot \vec{n} \frac{\vec{p}_{1} \cdot \vec{n}}{m_{1}}+6 \frac{\vec{S}_{1} \cdot \vec{p}_{2}}{m_{2}} \vec{S}_{2} \cdot \vec{n} \frac{\overrightarrow{p_{2}} \cdot \vec{n}}{m_{2}} \\
& +6 \vec{S}_{1} \cdot \vec{n} \frac{\vec{S}_{2} \cdot \vec{p}_{1}}{m_{1}} \frac{\vec{p}_{1} \cdot \vec{n}}{m_{1}}-\frac{21}{4} \vec{S}_{1} \cdot \vec{n} \frac{\vec{S}_{2} \cdot \vec{p}_{1}}{m_{1}} \frac{\vec{p}_{2} \cdot \vec{n}}{m_{2}}-\frac{9}{2} \vec{S}_{1} \cdot \vec{n} \frac{\vec{S}_{2} \cdot \vec{p}_{2}}{m_{2}} \frac{\vec{p}_{1} \cdot \vec{n}}{m_{1}} \\
& +\frac{3}{2} \vec{S}_{1} \cdot \vec{n} \frac{\vec{S}_{2} \cdot \vec{p}_{2}}{m_{2}} \frac{\vec{p}_{2} \cdot \vec{n}}{m_{2}}-\frac{3}{2} \vec{S}_{1} \cdot \vec{n} \vec{S}_{2} \cdot \vec{n} \frac{p_{1}^{2}}{m_{1}^{2}}+\frac{21}{4} \vec{S}_{1} \cdot \vec{n} \vec{S}_{2} \cdot \vec{n} \frac{\vec{p}_{1} \cdot \vec{p}_{2}}{m_{1} m_{2}} \\
& \left.-\frac{3}{2} \vec{S}_{1} \cdot \vec{n} \vec{S}_{2} \cdot \vec{n} \frac{p_{2}^{2}}{m_{2}^{2}}+\frac{15}{2} \vec{S}_{1} \cdot \vec{n} \vec{S}_{2} \cdot \vec{n} \frac{\vec{p}_{1} \cdot \vec{n}}{m_{1}} \frac{\vec{p}_{2} \cdot \vec{n}}{m_{2}}\right] \\
+ & \frac{G^{2}\left(m_{1}+m_{2}\right)}{r^{4}}\left[7 \vec{S}_{1} \cdot \vec{S}_{2}-13 \vec{S}_{1} \cdot \vec{n} \vec{S}_{2} \cdot \vec{n}\right] \cdot
\end{aligned}
$$




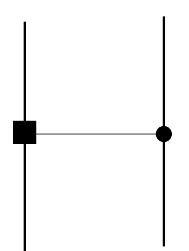

(a)

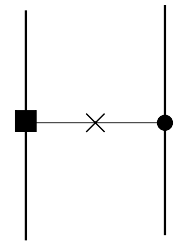

(b)

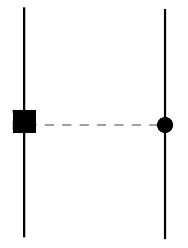

(c)

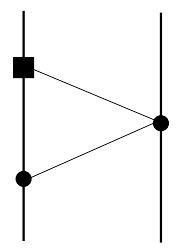

(d)

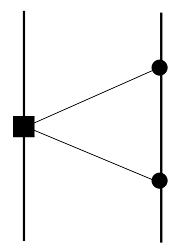

(e)

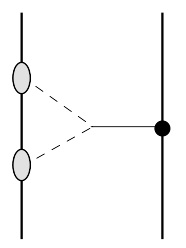

(f)

Figure 4. NLO spin-squared Feynman diagrams. These diagrams should be included together with their mirror images.

Again, to show equivalence with the ADM Hamiltonian in [69], we use the generator of canonical transformations in eq. (7.6) of [22] with the same notations. We find that the difference is resolved with the coefficients of the generator being set to

$$
g_{2}=1, \quad g_{3}=1,
$$

and the remaining coefficients set to zero.

\subsection{NLO spin-squared sector}

Finally, we compute the NLO spin-squared interaction, which was first approached in [38]. Here we treat it by means of the current EFT formulation for spin, and in terms of the NRG fields. Since the spin-squared coupling is actually no different than a mass quadrupole, it is in fact even more advantageous to use the NRG fields in this sector than in the linear in spin sectors, since similarly to the non-spinning point-mass sectors higher-loop diagrams are removed, e.g. as in the Einstein-Infeld-Hoffmann 1PN order potential, where a one-loop diagram is absent with the use of NRG fields [25].

We find that there are 6 Feynman diagrams in this sector shown in figure 4 . These diagrams are evaluated as follows:

$$
\begin{aligned}
& \text { Figure } 4(\mathrm{a})=\frac{C_{1\left(E S^{2}\right)}}{2} \frac{G m_{2}}{r^{3} m_{1}}\left[\frac{5}{2} S_{1}^{2} v_{1}^{2}+\frac{3}{2} S_{1}^{2} v_{2}^{2}-\left(\vec{S}_{1} \cdot \vec{v}_{1}\right)^{2}-3 S_{1}^{2}\left(\vec{v}_{1} \cdot \vec{n}\right)^{2}\right. \\
& \left.+3 \vec{S}_{1} \cdot \vec{v}_{1} \vec{S}_{1} \cdot \vec{n} \vec{v}_{1} \cdot \vec{n}-\frac{9}{2}\left(\vec{S}_{1} \cdot \vec{n}\right)^{2} v_{1}^{2}-\frac{9}{2}\left(\vec{S}_{1} \cdot \vec{n}\right)^{2} v_{2}^{2}\right] \\
& +C_{1\left(E S^{2}\right)} \frac{G m_{2}}{r^{2} m_{1}}\left[\vec{S}_{1} \cdot \vec{a}_{1} \vec{S}_{1} \cdot \vec{n}+\dot{\vec{S}}_{1} \cdot \vec{v}_{1} \vec{S}_{1} \cdot \vec{n}+\dot{\vec{S}}_{1} \cdot \vec{n} \vec{S}_{1} \cdot \vec{v}_{1}-\dot{S}_{1}^{2} \vec{v}_{1} \cdot \vec{n}\right] \\
& +C_{1\left(E S^{2}\right)} \frac{G m_{2}}{r m_{1}} \ddot{S}_{1}^{2},
\end{aligned}
$$

Figure $4(\mathrm{~b})=-\frac{C_{1\left(E S^{2}\right)}}{4} \frac{G m_{2}}{r^{3} m_{1}}\left[S_{1}^{2} \vec{v}_{1} \cdot \vec{v}_{2}-2 \vec{S}_{1} \cdot \vec{v}_{1} \vec{S}_{1} \cdot \vec{v}_{2}-3 S_{1}^{2} \vec{v}_{1} \cdot \vec{n} \overrightarrow{v_{2}} \cdot \vec{n}\right.$

$$
\begin{aligned}
& +6 \vec{S}_{1} \cdot \vec{v}_{1} \vec{S}_{1} \cdot \vec{n} \vec{v}_{2} \cdot \vec{n}+6 \vec{S}_{1} \cdot \vec{v}_{2} \vec{S}_{1} \cdot \vec{n} \vec{v}_{1} \cdot \vec{n}+3\left(\vec{S}_{1} \cdot \vec{n}\right)^{2} \vec{v}_{1} \cdot \vec{v}_{2} \\
& \left.\quad-15\left(\vec{S}_{1} \cdot \vec{n}\right)^{2} \vec{v}_{1} \cdot \vec{n} \vec{v}_{2} \cdot \vec{n}\right] \\
& +\frac{C_{1\left(E S^{2}\right)}}{4} \frac{G m_{2}}{r^{2} m_{1}}\left[2 \dot{\vec{S}}_{1} \cdot \vec{v}_{2} \vec{S}_{1} \cdot \vec{n}+2 \dot{\vec{S}}_{1} \cdot \vec{n} \vec{S}_{1} \cdot \vec{v}_{2}\right. \\
& \left.\quad-6 \dot{\vec{S}}_{1} \cdot \vec{n} \vec{S}_{1} \cdot \vec{n} \vec{v}_{2} \cdot \vec{n}-\dot{S}_{1}^{2} \vec{v}_{2} \cdot \vec{n}\right],
\end{aligned}
$$




$$
\begin{aligned}
\text { Figure } 4(\mathrm{c})= & -2 C_{1\left(E S^{2}\right)} \frac{G m_{2}}{r^{3} m_{1}}\left[S_{1}^{2}-3\left(\vec{S}_{1} \cdot \vec{n}\right)^{2}\right] \vec{v}_{1} \cdot \vec{v}_{2} \\
& -2 C_{1\left(E S^{2}\right)} \frac{G m_{2}}{r^{2} m_{1}}\left[\dot{\vec{S}}_{1} \cdot \vec{v}_{2} \vec{S}_{1} \cdot \vec{n}+\dot{\vec{S}}_{1} \cdot \vec{n} \vec{S}_{1} \cdot \vec{v}_{2}-\dot{S}_{1}^{2} \vec{v}_{2} \cdot \vec{n}\right], \\
\text { Figure 4(d) }= & -C_{1\left(E S^{2}\right)} \frac{G^{2} m_{2}}{2 r^{4}}\left[S_{1}^{2}-3\left(\vec{S}_{1} \cdot \vec{n}\right)^{2}\right], \\
\text { Figure 4(e) }= & -2 C_{1\left(E S^{2}\right)} \frac{G^{2}}{r^{4}} \frac{m_{2}^{2}}{m_{1}}\left[S_{1}^{2}-3\left(\vec{S}_{1} \cdot \vec{n}\right)^{2}\right], \\
\text { Figure 4(f) }= & \frac{G^{2} m_{2}}{r^{4}}\left(\vec{S}_{1} \cdot \vec{n}\right)^{2},
\end{aligned}
$$

where $\dot{S}_{1}^{2}=2 \dot{\vec{S}}_{1} \cdot \vec{S}_{1}$ is the time derivative of the spin length from eq. (4.5), and $\ddot{S}_{1}^{2}$ is its second time derivative. Note that due to the use of NRG fields, we do not have oneloop diagrams with the spin-squared coupling since as explained it is actually no different than a mass quadrupole. Thus, we do not have here diagrams similar to the two one-loop diagrams, which appear in figure 4(a), (b), of [38]. Therefore, the number of diagrams is reduced in this sector too, eliminating in particular those, which are more complicated to evaluate.

The potential. Summing all Feynman diagrams, we obtain the following potential:

$$
\begin{aligned}
V_{\mathrm{SS}}^{\mathrm{NLO}}=- & \frac{C_{1\left(E S^{2}\right)}}{2} \frac{G m_{2}}{r^{3} m_{1}}\left[\frac{5}{2} S_{1}^{2} v_{1}^{2}-\frac{9}{2} S_{1}^{2} \vec{v}_{1} \cdot \vec{v}_{2}+\frac{3}{2} S_{1}^{2} v_{2}^{2}-\left(\vec{S}_{1} \cdot \vec{v}_{1}\right)^{2}+\vec{S}_{1} \cdot \vec{v}_{1} \vec{S}_{1} \cdot \vec{v}_{2}\right. \\
& -3 S_{1}^{2}\left(\vec{v}_{1} \cdot \vec{n}\right)^{2}+\frac{3}{2} S_{1}^{2} \vec{v}_{1} \cdot \vec{n} \vec{v}_{2} \cdot \vec{n}+3 \vec{S}_{1} \cdot \vec{v}_{1} \vec{S}_{1} \cdot \vec{n} \vec{v}_{1} \cdot \vec{n}-3 \vec{S}_{1} \cdot \vec{v}_{1} \vec{S}_{1} \cdot \vec{n} \vec{v}_{2} \cdot \vec{n} \\
& -3 \vec{S}_{1} \cdot \vec{v}_{2} \vec{S}_{1} \cdot \vec{n} \vec{v}_{1} \cdot \vec{n}-\frac{9}{2}\left(\vec{S}_{1} \cdot \vec{n}\right)^{2} v_{1}^{2}+\frac{21}{2}\left(\vec{S}_{1} \cdot \vec{n}\right)^{2} \vec{v}_{1} \cdot \vec{v}_{2}-\frac{9}{2}\left(\vec{S}_{1} \cdot \vec{n}\right)^{2} v_{2}^{2} \\
& \left.+\frac{15}{2}\left(\vec{S}_{1} \cdot \vec{n}\right)^{2} \vec{v}_{1} \cdot \vec{n} \vec{v}_{2} \cdot \vec{n}\right]+\frac{C_{1\left(E S^{2}\right)}}{2} \frac{G^{2} m_{2}}{r^{4}}\left[S_{1}^{2}-3\left(\vec{S}_{1} \cdot \vec{n}\right)^{2}\right] \\
& +2 C_{1\left(E S^{2}\right)} \frac{G^{2} m_{2}^{2}}{r^{4} m_{1}}\left[S_{1}^{2}-3\left(\vec{S}_{1} \cdot \vec{n}\right)^{2}\right]-\frac{G^{2} m_{2}}{r^{4}}\left(\vec{S}_{1} \cdot \vec{n}\right)^{2} \\
& -C_{1\left(E S^{2}\right)} \frac{G m_{2}}{r^{2} m_{1}}\left[\vec{S}_{1} \cdot \vec{a}_{1} \vec{S}_{1} \cdot \vec{n}+\dot{\vec{S}}_{1} \cdot \vec{v}_{1} \vec{S}_{1} \cdot \vec{n}-\frac{3}{2} \dot{\vec{S}}_{1} \cdot \vec{v}_{2} \vec{S}_{1} \cdot \vec{n}+\dot{\vec{S}}_{1} \cdot \vec{n} \vec{S}_{1} \cdot \vec{v}_{1}\right. \\
& \left.\quad-\frac{3}{2} \dot{\vec{S}}_{1} \cdot \vec{n} \vec{S}_{1} \cdot \vec{v}_{2}-\frac{3}{2} \dot{\vec{S}}_{1} \cdot \vec{n} \vec{S}_{1} \cdot \vec{n} \vec{v}_{2} \cdot \vec{n}-\dot{S}_{1}^{2} \vec{v}_{1} \cdot \vec{n}+\frac{7}{4} \dot{S}_{1}^{2} \vec{v}_{2} \cdot \vec{n}\right] \\
& -C_{1\left(E S^{2}\right)} \frac{G m_{2}}{m_{1} r} \ddot{S}_{1}^{2}+1 \leftrightarrow 2 .
\end{aligned}
$$

We note that we can already take $\dot{S}^{2}=\ddot{S}^{2}=0$ since as we noted dissipative effects from the absorption of gravitational waves by the compact objects, which modify the spin length, are relevant only as of the $5 \mathrm{PN}$ order, and thus to our approximation the spin length $S^{2}$ is constant. After we make the shift of positions according to eq. (6.4), we get contributions linear in the shifts from the LO spin-orbit potential, equivalent to the insertion of EOM 
from this sector. This contribution reads

$$
\begin{aligned}
\Delta V_{\mathrm{SS}}^{\mathrm{NLO}}\left(\Delta \vec{y}_{I}\right)= & \frac{G m_{2}}{r^{3} m_{1}}\left[S_{1}^{2} v_{1}^{2}-S_{1}^{2} \vec{v}_{1} \cdot \vec{v}_{2}-\left(\vec{S}_{1} \cdot \vec{v}_{1}\right)^{2}+\vec{S}_{1} \cdot \vec{v}_{1} \vec{S}_{1} \cdot \vec{v}_{2}+3 \vec{S}_{1} \cdot \vec{v}_{1} \vec{S}_{1} \cdot \vec{n} \vec{v}_{1} \cdot \vec{n}\right. \\
& \left.-3 \vec{S}_{1} \cdot \vec{v}_{2} \vec{S}_{1} \cdot \vec{n} \vec{v}_{1} \cdot \vec{n}-3\left(\vec{S}_{1} \cdot \vec{n}\right)^{2} v_{1}^{2}+3\left(\vec{S}_{1} \cdot \vec{n}\right)^{2} \vec{v}_{1} \cdot \vec{v}_{2}\right] \\
& -\frac{1}{2 m_{1}}\left[S_{1}^{2} \dot{\vec{a}}_{1} \cdot \vec{v}_{1}-\vec{S}_{1} \cdot \dot{\vec{a}}_{1} \vec{S}_{1} \cdot \vec{v}_{1}\right]+1 \leftrightarrow 2 .
\end{aligned}
$$

Finally, we note that there is an addition to this sector also from terms quadratic in the shift in eq. (6.4), originating from the Newtonian sector. It is given by

$$
\begin{gathered}
\Delta V_{\mathrm{SS}}^{\mathrm{NLO}}\left(\left(\Delta \vec{y}_{I}\right)^{2}\right)=-\frac{G m_{2}}{8 r^{3} m_{1}}\left[2 S_{1}^{2} v_{1}^{2}-2\left(\vec{S}_{1} \cdot \vec{v}_{1}\right)^{2}-3 S_{1}^{2}\left(\vec{v}_{1} \cdot \vec{n}\right)^{2}+6 \vec{S}_{1} \cdot \vec{v}_{1} \vec{S}_{1} \cdot \vec{n} \vec{v}_{1} \cdot \vec{n}\right. \\
\left.-3\left(\vec{S}_{1} \cdot \vec{n}\right)^{2} v_{1}^{2}\right]-\frac{1}{8 m_{1}}\left[S_{1}^{2} a_{1}^{2}-\left(\vec{S}_{1} \cdot \vec{a}_{1}\right)^{2}\right]+1 \leftrightarrow 2 .
\end{gathered}
$$

After this shift, we proceed to eliminate the remaining higher order time derivatives, including time derivatives of spin by insertion of the LO Newtonian EOM of the spin. For completeness we present the explicit NLO redefinition of position, which removes its higher order time derivatives. It reads

$$
\vec{y}_{1} \rightarrow \vec{y}_{1}+C_{1\left(E S^{2}\right)} \frac{G m_{2}}{m_{1}^{2} r^{2}} \vec{S}_{1} \vec{S}_{1} \cdot \vec{n}+\frac{G m_{2}}{8 m_{1}^{2} r^{2}}\left(\vec{S}_{1} \vec{S}_{1} \cdot \vec{n}-\vec{n} S_{1}^{2}\right)+\frac{3}{8 m_{1}^{2}}\left(\vec{S}_{1} \vec{S}_{1} \cdot \vec{a}_{1}-\vec{a}_{1} S_{1}^{2}\right),
$$

and similarly for particle 2 with $1 \leftrightarrow 2$. The contribution to the action, linear in this shift, removes the acceleration terms, and is equivalent to substituting in the EOM of the position. For the explicit redefinition of spin we have, using the antisymmetric generator of rotation $\omega_{1}^{i j}$, that

$$
\begin{aligned}
\omega_{1}^{i j}= & C_{1\left(E S^{2}\right)} \frac{G m_{2}}{m_{1} r^{2}}\left(\left(-S_{1}^{i k} v_{1}^{j} n^{k}-S_{1}^{i k} n^{j} v_{1}^{k}+\frac{3}{2} S_{1}^{i k} v_{2}^{j} n^{k}+\frac{3}{2} S_{1}^{i k} n^{j} v_{2}^{k}+\frac{3}{2} S_{1}^{i k} n^{j} n^{k} \vec{v}_{2} \cdot \vec{n}\right)\right. \\
& \left.-(i \leftrightarrow j)+2 S_{1}^{i j} \vec{v}_{1} \cdot \vec{n}-3 S_{1}^{i j} \vec{v}_{2} \cdot \vec{n}\right)-\frac{G m_{2}}{4 m_{1} r^{2}}\left(S_{1}^{i k} v_{1}^{j} n^{k}-S_{1}^{i k} n^{j} v_{1}^{k}-(i \leftrightarrow j)\right) \\
& -\frac{1}{4 m_{1}}\left(S_{1}^{i k} v_{1}^{j} a_{1}^{k}+S_{1}^{i k} a_{1}^{j} v_{1}^{k}-(i \leftrightarrow j)\right)
\end{aligned}
$$

and similarly for particle 2 with $1 \leftrightarrow 2$. The contribution to the action, linear in this shift, removes the precession terms, and is equivalent to substituting in the EOM of the spin. Note that the redefinitions in eqs. (6.43) and (6.44) contain terms with accelerations. These terms are required in order to remove from the potential terms, which are quadratic in the accelerations.

The Hamiltonian. Again, one can perform a straightforward Legendre transform to obtain the Hamiltonian:

$$
\begin{aligned}
H_{\mathrm{SS}}^{\mathrm{NLO}}=- & \frac{C_{1\left(E S^{2}\right)}}{2} \frac{G m_{2}}{r^{3} m_{1}}\left[\frac{5}{2} S_{1}^{2} \frac{p_{1}^{2}}{m_{1}^{2}}-\frac{9}{2} S_{1}^{2} \frac{\vec{p}_{1} \cdot \vec{p}_{2}}{m_{1} m_{2}}+\frac{3}{2} S_{1}^{2} \frac{p_{2}^{2}}{m_{2}^{2}}-\left(\frac{\vec{S}_{1} \cdot \vec{p}_{1}}{m_{1}}\right)^{2}\right. \\
& +\frac{\vec{S}_{1} \cdot \vec{p}_{1}}{m_{1}} \frac{\vec{S}_{1} \cdot \vec{p}_{2}}{m_{2}}-3 S_{1}^{2}\left(\frac{\vec{p}_{1} \cdot \vec{n}}{m_{1}}\right)^{2}+\frac{3}{2} S_{1}^{2} \frac{\vec{p}_{1} \cdot \vec{n}}{m_{1}} \frac{\vec{p}_{2} \cdot \vec{n}}{m_{2}}+3 \frac{\vec{S}_{1} \cdot \vec{p}_{1}}{m_{1}} \vec{S}_{1} \cdot \vec{n} \frac{\overrightarrow{p_{1}} \cdot \vec{n}}{m_{1}}
\end{aligned}
$$




$$
\begin{aligned}
& -3 \frac{\vec{S}_{1} \cdot \vec{p}_{1}}{m_{1}} \vec{S}_{1} \cdot \vec{n} \frac{\vec{p}_{2} \cdot \vec{n}}{m_{2}}-3 \frac{\vec{S}_{1} \cdot \vec{p}_{2}}{m_{2}} \vec{S}_{1} \cdot \vec{n} \frac{\vec{p}_{1} \cdot \vec{n}}{m_{1}}-\frac{9}{2}\left(\vec{S}_{1} \cdot \vec{n}\right)^{2} \frac{p_{1}^{2}}{m_{1}^{2}} \\
& \left.+\frac{21}{2}\left(\vec{S}_{1} \cdot \vec{n}\right)^{2} \frac{\vec{p}_{1} \cdot \vec{p}_{2}}{m_{1} m_{2}}-\frac{9}{2}\left(\vec{S}_{1} \cdot \vec{n}\right)^{2} \frac{p_{2}^{2}}{m_{2}^{2}}+\frac{15}{2}\left(\vec{S}_{1} \cdot \vec{n}\right)^{2} \frac{\vec{p}_{1} \cdot \vec{n}}{m_{1}} \frac{\overrightarrow{p_{2}} \cdot \vec{n}}{m_{2}}\right] \\
& +\frac{G m_{2}}{r^{3} m_{1}}\left[\frac{5}{4} S_{1}^{2} \frac{p_{1}^{2}}{m_{1}^{2}}-\frac{3}{2} S_{1}^{2} \frac{\vec{p}_{1} \cdot \vec{p}_{2}}{m_{1} m_{2}}-\frac{5}{4}\left(\frac{\vec{S}_{1} \cdot \vec{p}_{1}}{m_{1}}\right)^{2}+\frac{3}{2} \frac{\vec{S}_{1} \cdot \vec{p}_{1}}{m_{1}} \frac{\vec{S}_{1} \cdot \vec{p}_{2}}{m_{2}}\right. \\
& -\frac{9}{8} S_{1}^{2}\left(\frac{\vec{p}_{1} \cdot \vec{n}}{m_{1}}\right)^{2}+\frac{3}{2} S_{1}^{2} \frac{\vec{p}_{1} \cdot \vec{n}}{m_{1}} \frac{\overrightarrow{p_{2}} \cdot \vec{n}}{m_{2}}+\frac{15}{4} \frac{\vec{S}_{1} \cdot \vec{p}_{1}}{m_{1}} \vec{S}_{1} \cdot \vec{n} \frac{\overrightarrow{p_{1}} \cdot \vec{n}}{m_{1}} \\
& -\frac{3}{2} \frac{\vec{S}_{1} \cdot \vec{p}_{1}}{m_{1}} \vec{S}_{1} \cdot \vec{n} \frac{\vec{p}_{2} \cdot \vec{n}}{m_{2}}-3 \frac{\vec{S}_{1} \cdot \vec{p}_{2}}{m_{2}} \vec{S}_{1} \cdot \vec{n} \frac{\vec{p}_{1} \cdot \vec{n}}{m_{1}}-\frac{21}{8}\left(\vec{S}_{1} \cdot \vec{n}\right)^{2} \frac{p_{1}^{2}}{m_{1}^{2}} \\
& \left.+3\left(\vec{S}_{1} \cdot \vec{n}\right)^{2} \frac{\vec{p}_{1} \cdot \vec{p}_{2}}{m_{1} m_{2}}\right] \\
& +\frac{C_{1\left(E S^{2}\right)}}{2} \frac{G^{2} m_{2}}{r^{4}}\left[S_{1}^{2}-3\left(\vec{S}_{1} \cdot \vec{n}\right)^{2}\right]+C_{1\left(E S^{2}\right)} \frac{G^{2} m_{2}^{2}}{r^{4} m_{1}}\left[2 S_{1}^{2}-5\left(\vec{S}_{1} \cdot \vec{n}\right)^{2}\right] \\
& +\frac{G^{2} m_{2}}{r^{4}}\left[2 S_{1}^{2}-3\left(\vec{S}_{1} \cdot \vec{n}\right)^{2}\right]+\frac{G^{2} m_{2}^{2}}{r^{4} m_{1}}\left[S_{1}^{2}-\left(\vec{S}_{1} \cdot \vec{n}\right)^{2}\right]+1 \leftrightarrow 2 .
\end{aligned}
$$

For the NLO spin-squared sector we need to construct the general infinitesimal generator of canonical transformations in order to show equivalence with the ADM Hamiltonian in [70]. Similar to the considerations in section 7 of [22], we have the following general form for this generator:

$$
\begin{aligned}
g_{\mathrm{SS}}^{\mathrm{NLO}}=\frac{G m_{2}}{r^{2} m_{1}}\left[S_{1}^{2}\right. & \left(g_{1} \frac{\vec{p}_{1} \cdot \vec{n}}{m_{1}}+g_{2} \frac{\vec{p}_{2} \cdot \vec{n}}{m_{2}}\right)+\vec{S}_{1} \cdot \vec{n}\left(g_{3} \frac{\vec{S}_{1} \cdot \vec{p}_{1}}{m_{1}}+g_{4} \frac{\vec{S}_{1} \cdot \vec{p}_{2}}{m_{2}}\right) \\
& \left.+\left(\vec{S}_{1} \cdot \vec{n}\right)^{2}\left(g_{5} \frac{\overrightarrow{p_{1}} \cdot \vec{n}}{m_{1}}+g_{6} \frac{\overrightarrow{p_{2}} \cdot \vec{n}}{m_{2}}\right)\right],
\end{aligned}
$$

where this generator should be taken with $1 \leftrightarrow 2$. We find then that the difference of Hamiltonians is resolved with the coefficients of the generator fixed to

$$
g_{3}=C_{1\left(E S^{2}\right)},
$$

and the remaining ones fixed to zero.

\section{Conclusions}

In this paper we have presented a formulation for gravitating spinning objects in the effective field theory in the post-Newtonian scheme. We aimed to attain accuracy at the $4 \mathrm{PN}$ order for rapidly rotating compact objects, and indeed the formulation holds as it stands to this high PN order. Such high PN orders are required for the successful detection of gravitational radiation.

A crucial aspect in our EFT formulation for spinning objects is that we indeed eventually obtain an effective action, where all field modes below the orbital scale are integrated out. This is achieved by introducing several new ingredients, on which this work strongly 
builds. First, we point out the relevant degrees of freedom, taking special notice of the rotational ones, and most importantly the associated symmetries. Building on these symmetries, we introduce the minimal coupling part of the point particle action in terms of gauge rotational variables, and construct the spin-induced nonminimal couplings, where we obtain the LO couplings to all orders in spin. We then introduce the gauge freedom of rotational variables into the point particle action. Altogether, we construct the point particle effective action following symmetry considerations for spinning objects for the first time. Finally, we fix a canonical gauge for the rotational variables, where the unphysical DOFs are eliminated already from the Feynman rules, and all the orbital field modes are conveniently integrated out.

The EOM of spin are then directly obtained via a proper variation of the action, where they take on a simple form. Moreover, the corresponding Hamiltonians are also straightforwardly obtained from the potentials derived via this formulation, due to the canonical gauge fixing of the rotational variables. The EFT formulation for spin is implemented here to derive all spin dependent potentials up to NLO to quadratic level in spin, i.e. up to the 3PN order for rapidly rotating compact objects. In particular, proper NLO spin-squared potential and Hamiltonian are also derived. For these implementations we use the NRG field decomposition, which is found to eliminate higher-loop Feynman diagrams also in the spin dependent sectors, and facilitates derivations. Therefore, with the additional advantageous usefulness of the obtained Hamiltonians, which relate to GW observables, the EFT formulation for spin here is ideal for the treatment of higher order spin dependent sectors.

In order to complete the spin dependent conservative sector, i.e. potentials to $4 \mathrm{PN}$ order, it remains to apply this EFT formulation for spin at NNLO up to quadratic level in spin, which was initiated in [67]. Indeed, this was recently obtained in [29], and [30], for the spin-orbit, and spin-squared sectors, respectively, in addition to the LO cubic and quartic in spin sector in [28]. Further, one may proceed to obtain a formulation of an EFT of radiation for spin for the radiative sector along the lines of this work. Implementation on the radiative sector covering up to $4 \mathrm{PN}$ order would then follow. Finally, it is left for future research to reach a better physical understanding of the various gauges of the rotational variables, and of the extra term from minimal coupling, which arises from introducing the spin gauge freedom in the action, and contributes to finite size effects with spin.

\section{Acknowledgments}

This work has been done within the Labex ILP (reference ANR-10-LABX-63) part of the Idex SUPER, and received financial French state aid managed by the Agence Nationale de la Recherche, as part of the programme Investissements d'Avenir under the reference ANR-11-IDEX-0004-02.

Open Access. This article is distributed under the terms of the Creative Commons Attribution License (CC-BY 4.0), which permits any use, distribution and reproduction in any medium, provided the original author(s) and source are credited. 


\section{References}

[1] LIGO webpage, http://www.ligo.caltech.edu.

[2] Virgo webpage, https://wwwcascina.virgo.infn.it.

[3] KAGRA webpage, http://gwcenter.icrr.u-tokyo.ac.jp/en.

[4] ESA LISA webpage, http://sci.esa.int/lisa.

[5] eLISA webpage, https://www.elisascience.org.

[6] L. Blanchet, Gravitational radiation from post-newtonian sources and inspiralling compact binaries, Living Rev. Rel. 17 (2014) 2 [arXiv:1310.1528] [InSPIRE].

[7] K. Yagi and N. Yunes, Love can be tough to measure, Phys. Rev. D 89 (2014) 021303 [arXiv:1310.8358] [INSPIRE].

[8] J.E. McClintock et al., Measuring the spins of accreting black holes, Class. Quant. Grav. 28 (2011) 114009 [arXiv:1101.0811] [InSPIRE].

[9] T. Damour, P. Jaranowski and G. Schäfer, Nonlocal-in-time action for the fourth post-Newtonian conservative dynamics of two-body systems, Phys. Rev. D 89 (2014) 064058 [arXiv: 1401.4548] [INSPIRE].

[10] A.J. Hanson and T. Regge, The relativistic spherical top, Annals Phys. 87 (1974) 498 [INSPIRE].

[11] I. Bailey and W. Israel, Lagrangian dynamics of spinning particles and polarized media in general relativity, Commun. Math. Phys. 42 (1975) 65.

[12] W.D. Goldberger and I.Z. Rothstein, An effective field theory of gravity for extended objects, Phys. Rev. D 73 (2006) 104029 [hep-th/0409156] [InSPIRE].

[13] W.D. Goldberger, Les Houches lectures on effective field theories and gravitational radiation, hep-ph/0701129 [INSPIRE].

[14] T. Damour and G. Esposito-Farese, Gravitational wave versus binary - Pulsar tests of strong field gravity, Phys. Rev. D 58 (1998) 042001 [gr-qc/9803031] [InSPIRE].

[15] T. Damour and G. Esposito-Farese, Testing gravity to second postNewtonian order: a field theory approach, Phys. Rev. D 53 (1996) 5541 [gr-qc/9506063] [INSPIRE].

[16] R.A. Porto, Post-Newtonian corrections to the motion of spinning bodies in NRGR, Phys. Rev. D 73 (2006) 104031 [gr-qc/0511061] [INSPIRE].

[17] R.A. Porto and I.Z. Rothstein, Spin(1)Spin(2) effects in the motion of inspiralling compact binaries at third order in the post-Newtonian expansion, Phys. Rev. D 78 (2008) 044012 [Erratum ibid. D 81 (2010) 029904] [arXiv: 0802.0720] [INSPIRE].

[18] K. Yee and M. Bander, Equations of motion for spinning particles in external electromagnetic and gravitational fields, Phys. Rev. D 48 (1993) 2797 [hep-th/9302117] [InSPIRE].

[19] M. Levi, Next to leading order gravitational Spin(1)-Spin(2) coupling with Kaluza-Klein reduction, Phys. Rev. D 82 (2010) 064029 [arXiv:0802.1508] [InSPIRE].

[20] M. Levi, Next to leading order gravitational spin-orbit coupling in an effective field theory approach, Phys. Rev. D 82 (2010) 104004 [arXiv:1006.4139] [INSPIRE]. 
[21] S. Hergt, J. Steinhoff and G. Schäfer, Elimination of the spin supplementary condition in the effective field theory approach to the post-Newtonian approximation, Annals Phys. 327 (2012) 1494 [arXiv:1110.2094] [INSPIRE].

[22] M. Levi and J. Steinhoff, Equivalence of ADM Hamiltonian and Effective Field Theory approaches at next-to-next-to-leading order Spin(1)-Spin(2) coupling of binary inspirals, JCAP 12 (2014) 003 [arXiv: 1408.5762] [INSPIRE].

[23] W. Tulczyjew, Equations of motion of rotating bodies in general relativity theory, Acta Phys. Polon. 18 (1959) 37 [Erratum ibid. 18 (1959) 534].

[24] E. Poisson, Absorption of mass and angular momentum by a black hole: Time-domain formalisms for gravitational perturbations and the small-hole/slow-motion approximation, Phys. Rev. D 70 (2004) 084044 [gr-qc/0407050] [INSPIRE].

[25] B. Kol and M. Smolkin, Non-relativistic gravitation: from Newton to Einstein and back, Class. Quant. Grav. 25 (2008) 145011 [arXiv:0712.4116] [INSPIRE].

[26] B. Kol, M. Levi and M. Smolkin, Comparing space+time decompositions in the post-Newtonian limit, Class. Quant. Grav. 28 (2011) 145021 [arXiv:1011.6024] [INSPIRE].

[27] A. Buonanno and T. Damour, Effective one-body approach to general relativistic two-body dynamics, Phys. Rev. D 59 (1999) 084006 [gr-qc/9811091] [INSPIRE].

[28] M. Levi and J. Steinhoff, Leading order finite size effects with spins for inspiralling compact binaries, JHEP 06 (2015) 059 [arXiv:1410.2601] [INSPIRE].

[29] M. Levi and J. Steinhoff, Next-to-next-to-leading order gravitational spin-orbit coupling via the effective field theory for spinning objects in the post-Newtonian scheme, arXiv:1506.05056 [INSPIRE].

[30] M. Levi and J. Steinhoff, Next-to-next-to-leading order gravitational spin-squared potential via the effective field theory for spinning objects in the post-Newtonian scheme, arXiv: 1506.05794 [INSPIRE].

[31] T. Appelquist and J. Carazzone, Infrared singularities and massive fields, Phys. Rev. D 11 (1975) 2856 [inSPIRE].

[32] W. Tulczyjew, Motion of multipole particles in general relativity theory, Acta Phys. Polon. 18 (1959) 393.

[33] R. Schattner, The center-of-mass in general relativity, Gen. Rel. Grav. 10 (1979) 377 [INSPIRE].

[34] R. Schattner, The uniqueness of the center-of-mass in general relativity, Gen. Rel. Grav. 10 (1979) 395 [inSPIRE].

[35] D.E. Fahnline, Manifestly covariant, coordinate-free dyadic expression for planar homogeneous Lorentz transformations, J. Math. Phys. 24 (1983) 1080.

[36] J. Steinhoff, Spin gauge symmetry in the action principle for classical relativistic particles, arXiv: 1501.04951 [INSPIRE].

[37] T. Damour and G. Schaefer, Redefinition of position variables and the reduction of higher order Lagrangians, J. Math. Phys. 32 (1991) 127 [INSPIRE].

[38] R.A. Porto and I.Z. Rothstein, Next to leading order Spin(1)Spin(1) effects in the motion of inspiralling compact binaries, Phys. Rev. D 78 (2008) 044013 [Erratum ibid. D 81 (2010) 029905] [arXiv: 0804.0260] [INSPIRE]. 
[39] M. Mathisson, Neue mechanik materieller systemes, Acta Phys. Polon. 6 (1937) 163 [INSPIRE].

[40] M. Mathisson, Republication of: New mechanics of material systems, Gen. Rel. Grav. 42 (2010) 1011.

[41] A. Papapetrou, Spinning test particles in general relativity. 1, Proc. Roy. Soc. Lond. A 209 (1951) 248 [inSPIRE].

[42] W.D. Goldberger and I.Z. Rothstein, Dissipative effects in the worldline approach to black hole dynamics, Phys. Rev. D 73 (2006) 104030 [hep-th/0511133] [INSPIRE].

[43] W.D. Goldberger and A. Ross, Gravitational radiative corrections from effective field theory, Phys. Rev. D 81 (2010) 124015 [arXiv:0912.4254] [INSPIRE].

[44] S. Chakrabarti, T. Delsate and J. Steinhoff, Effective action and linear response of compact objects in Newtonian gravity, Phys. Rev. D 88 (2013) 084038 [arXiv:1306.5820] [INSPIRE].

[45] S. Chakrabarti, T. Delsate and J. Steinhoff, New perspectives on neutron star and black hole spectroscopy and dynamic tides, arXiv:1304.2228 [INSPIRE].

[46] H. Georgi, Effective field theory, Ann. Rev. Nucl. Part. Sci. 43 (1993) 209 [inSPIRE].

[47] S. Weinberg, Gravitation and cosmology: principles and applications of the general theory of relativity, Wiley, New York U.S.A. (1972).

[48] S. Weinberg, The quantum theory of fields. Volume 1: foundations, Cambridge University Press, Cambridge U.K. (1995).

[49] D. Bini, T. Damour and G. Faye, Effective action approach to higher-order relativistic tidal interactions in binary systems and their effective one body description, Phys. Rev. D 85 (2012) 124034 [arXiv:1202.3565] [INSPIRE].

[50] W.G. Laarakkers and E. Poisson, Quadrupole moments of rotating neutron stars, Astrophys. J. 512 (1999) 282 [gr-qc/9709033] [INSPIRE].

[51] G. Pappas and T.A. Apostolatos, Revising the multipole moments of numerical spacetimes and its consequences, Phys. Rev. Lett. 108 (2012) 231104 [arXiv:1201.6067] [INSPIRE].

[52] K. Yagi, K. Kyutoku, G. Pappas, N. Yunes and T.A. Apostolatos, Effective no-hair relations for neutron stars and quark stars: relativistic results, Phys. Rev. D 89 (2014) 124013 [arXiv: 1403.6243] [INSPIRE].

[53] B.M. Barker and R.F. O'Connell, Gravitational two-body problem with arbitrary masses, spins and quadrupole moments, Phys. Rev. D 12 (1975) 329 [INSPIRE].

[54] E. Poisson, Gravitational waves from inspiraling compact binaries: the quadrupole moment term, Phys. Rev. D 57 (1998) 5287 [gr-qc/9709032] [INSPIRE].

[55] S. Marsat, Cubic order spin effects in the dynamics and gravitational wave energy flux of compact object binaries, Class. Quant. Grav. 32 (2015) 085008 [arXiv:1411.4118] [InSPIRE].

[56] G. Faye, L. Blanchet and A. Buonanno, Higher-order spin effects in the dynamics of compact binaries. I. Equations of motion, Phys. Rev. D 74 (2006) 104033 [gr-qc/0605139] [INSPIRE].

[57] S. Marsat, A. Bohe, G. Faye and L. Blanchet, Next-to-next-to-leading order spin-orbit effects in the equations of motion of compact binary systems, Class. Quant. Grav. 30 (2013) 055007 [arXiv: 1210.4143] [INSPIRE]. 
[58] A. Bohe, S. Marsat, G. Faye and L. Blanchet, Next-to-next-to-leading order spin-orbit effects in the near-zone metric and precession equations of compact binaries,

Class. Quant. Grav. 30 (2013) 075017 [arXiv: 1212.5520] [INSPIRE].

[59] T. Damour, P. Jaranowski and G. Schaefer, Hamiltonian of two spinning compact bodies with next-to-leading order gravitational spin-orbit coupling, Phys. Rev. D 77 (2008) 064032 [arXiv:0711.1048] [INSPIRE].

[60] J. Steinhoff and G. Schaefer, Canonical formulation of self-gravitating spinning-object systems, Europhys. Lett. 87 (2009) 50004 [arXiv:0907.1967] [INSPIRE].

[61] T. Damour, M. Soffel and C.-m. Xu, General relativistic celestial mechanics. 1. Method and definition of reference systems, Phys. Rev. D 43 (1991) 3272 [INSPIRE].

[62] J.S. Schwinger, Quantized gravitational field, Phys. Rev. 130 (1963) 1253 [INSPIRE].

[63] M.H.L. Pryce, The mass center in the restricted theory of relativity and its connection with the quantum theory of elementary particles, Proc. Roy. Soc. Lond. A 195 (1948) 62 [INSPIRE].

[64] T.D. Newton and E.P. Wigner, Localized states for elementary systems, Rev. Mod. Phys. 21 (1949) 400 [inSPIRE].

[65] E. Barausse, E. Racine and A. Buonanno, Hamiltonian of a spinning test-particle in curved spacetime, Phys. Rev. D 80 (2009) 104025 [Erratum ibid. D 85 (2012) 069904] [arXiv: 0907.4745] [INSPIRE].

[66] E. Corinaldesi and A. Papapetrou, Spinning test particles in general relativity. 2, Proc. Roy. Soc. Lond. A 209 (1951) 259 [InSPIRE].

[67] M. Levi, Binary dynamics from Spin(1)-Spin(2) coupling at fourth post-Newtonian order, Phys. Rev. D 85 (2012) 064043 [arXiv:1107.4322] [InSPIRE].

[68] H. Tagoshi, A. Ohashi and B.J. Owen, Gravitational field and equations of motion of spinning compact binaries to 2.5 post-Newtonian order, Phys. Rev. D 63 (2001) 044006 [gr-qc/0010014] [INSPIRE].

[69] J. Steinhoff, G. Schaefer and S. Hergt, ADM canonical formalism for gravitating spinning objects, Phys. Rev. D 77 (2008) 104018 [arXiv:0805.3136] [INSPIRE].

[70] S. Hergt, J. Steinhoff and G. Schaefer, Reduced hamiltonian for next-to-leading order spin-squared dynamics of general compact binaries, Class. Quant. Grav. 27 (2010) 135007 [arXiv: 1002.2093] [INSPIRE]. 